\title{
Article \\ Effect of Helix Angle on the Performance of Helical Vertical Axis Wind Turbine
}

\author{
Unnikrishnan Divakaran ${ }^{1}$ (D) Ajith Ramesh ${ }^{1}$, Akram Mohammad ${ }^{2}$ and Ratna Kishore Velamati ${ }^{1, *}$ D \\ 1 Department of Mechanical Engineering, Amrita School of Engineering, Amrita Vishwa Vidyapeetham, \\ Coimbatore 641112, India; d_unnikrishnan@cb.amrita.edu (U.D.); r_ajith@cb.amrita.edu (A.R.) \\ 2 Department of Aeronautical Engineering, Faculty of Engineeirng, King Abdulaziz University, Jeddah 21589, \\ Saudi Arabia; ammohamad4@kau.edu.sa \\ * Correspondence: v_ratnakishore@cb.amrita.edu
}

Citation: Divakaran, U.; Ramesh, A.; Mohammad, A.; Velamati, R.K. Effect of Helix Angle on the Performance of Helical Vertical Axis Wind Turbine.

Energies 2021, 14, 393.

https://doi.org/10.3390/en14020393

Received: 26 November 2020

Accepted: 8 January 2021

Published: 12 January 2021

Publisher's Note: MDPI stays neutral with regard to jurisdictional clai$\mathrm{ms}$ in published maps and institutional affiliations.

Copyright: () 2021 by the authors. Licensee MDPI, Basel, Switzerland. This article is an open access article distributed under the terms and conditions of the Creative Commons Attribution (CC BY) license (https:// creativecommons.org/licenses/by/ $4.0 /)$.

\begin{abstract}
The energy crisis has forced researchers to look for various non-conventional energy sources. Wind energy is one of the potential sources, and researchers have invested resources in developing different kinds of wind turbines. Vertical axis wind turbines (VAWT) have received less attention than their horizontal-axis counterparts. A helical-bladed VAWT is preferred because it makes perfect sense as an improvement in design, as they have higher azimuth angles of power generation capabilities. This paper studies the effects of the helix angle of blades in the aerodynamic performance of VAWT using 3D numerical simulations. Three different helix angles of $60^{\circ}, 90^{\circ}$, and $120^{\circ}$ of a three-bladed VAWT operating across different tip speed ratios were studied. Turbulence is modelled using a four-equation transition SST k- $\omega$ model (shear stress transport). The $60^{\circ}$ helicalbladed VAWT was found to be better performing in comparison with all other helical-bladed and straight-bladed VAWT. The ripple effects on the shaft are also analysed using a standard deviation plot of the moment coefficient generated by a single blade over one complete cycle of its rotation. It was observed that the greater the helix angle, the lower the standard deviation. The paper also tries to analyse the percentage of power generated by each quartile of flow and the contribution of each section of the blade. Ansys FLUENT was employed for the entire study. A comparative study between different helical-bladed VAWT and straight-bladed VAWT was carried out along with wake structure analysis and flow contours for a better understanding of the flow field.
\end{abstract}

Keywords: vertical axis wind turbine; wind energy; helical blade; CFD

\section{Introduction}

Unhindered access to electric power is one of the most important factors ensuring the dynamic development of the countries and civilisations. Since the start of 20th century, fossil fuels have been considered as the most common energy carrier, which has caused a rapid growth of civilisation. However, due to ecological and economic issues, many countries are introducing new regulations to cut down the utilisation of fossil fuels and emission of $\mathrm{CO}_{2}$ [1-3]. According to these regulations, most of the states have committed to increase their fraction of the generation of the renewable sources of energy of the total installed electric power generation capacity. The wind energy can be a good alternative for fossil fuels, as it can help reduce the consumption of fossil fuels and emission of flue gases. The main disadvantage of using wind energy is its high sensitivity to the forecast conditions [4,5]. This disadvantage makes it difficult to control and foresee wind energy production. Other disadvantages are noise generated by wind turbines [6] as well as their visual [7] and environmental [8] impact (behaviour of animals, especially birds) on the surrounding. Some of the above problems can be overcome by decentralised application of vertical axis wind turbines (VAWTs), which are less sensitive to the wind conditions and have a broader range of operational wind speed. There are certain studies that prove 
that the performance of VAWTs are not limited to Betz number [9,10]; hence, units with efficiency higher than traditional horizontal axis wind turbines may be realised.

VAWTs are more suitable to be used in an urban environment because of the following reasons: lower noise emission, higher cut-off wind speed, lower minimum operational wind speed, low susceptibility to wind turbulence, omnidirectionality, and compact construction [11,12]. As a result of these reasons, there is growing interest for research (experimental and numerical) in VAWTs. However, there is still a lot to explore to attain the level of development comparable to the HAWTs.

VAWTs are broadly classified based on their prime motive force: lift (Darrieus turbines) and drag (Savonious turbines). Savoniuos turbines are suitable for low tip speed ratio (TSR) conditions and better self-starting, but their power coefficient is low when compared with similar Darrius turbines. On the other hand, Darrieus VAWTs are better performing at higher TSRs and are unaffected by lateral winds but have self-starting issues and structural loading problems due to cyclic forces. Different blade designs employed in lift-based VAWTs are straight, Troposkien (egg-beater shape), helical, canted, tulip shaped, and butterfly shaped, to name a few. These non-straight blade designs propose to improve the structural issues of the turbine.

The fluid flow around an operating lift-based VAWT is complex. There are a couple of phenomena that co-occur such as dynamic stall, vortex shedding, and flow over an airfoil with continuously changing attack angle. Understanding these phenomena would help to comprehend the aerodynamics of the turbine and thereby improve its performance. The geometric parameters such as solidity, pitch angle, and airfoil shape, and operational parameters such as the Reynolds number, turbulence intensity, and TSR affect the turbine performance. Various researchers of straight blade VAWT have extensively studied geometric parameters such as solidity [13-16], pitch angle [17-19], and airfoil shape [20-22]. Similarly, the operational parameters of a straight blade VAWT such as TSR $[23,24]$, turbulence intensity $[25,26]$, and Reynolds number of operation $[15,24,27]$ have been comprehensively studied also.

Recently, research in non-straight blade VAWT has been steadily growing. Blade shapes such as helical [28-30], Troposkien [31-35], V-shaped [36], and hybrid [37] have been studied by various researchers for their structural integrity. However, studies on the effect of these geometrical parameters on the aerodynamic performance of non-straightbladed VAWTs are limited. An investigation by Battisti et al. [31] on the numerical and experimental study of straight blade and Troposkien blade turbines of a similar swept area resulted in benchmarking the performance of these turbines. The review, which was mostly about the performance of the small-scale turbines in different operating conditions, resulted in a better understanding of how the flow features affected the incoming blade. Lee and Lim [38] in their numerical study of VAWT (chord of $150 \mathrm{~mm}$ ) (height $600 \mathrm{~mm}$ ) (rotor diameter of $740 \mathrm{~mm}$ ) with varying helical angles $\left(0^{\circ}\right.$ to $\left.30^{\circ}\right)$, concluded that the helix angle does not improve the performance of the turbine. In another study by Scheurich [39] on the effect of blade curvature and helix angle, it was concluded that for a better understanding of the aerodynamic effects of a non-straight blade, it is essential to analyse the interactions of wakes of the non-straight blade of a VAWT. A numerical study on part blade VAWT in comparison to helical VAWT by Karimian and Abdolahifar [40] focussed on the effect of segmented blades that perform similar to the helical blade. The study on the performance characterisation of these turbines operating under different TSRs concluded by defending the three-part blade model to be more effective at lower TSR, giving better self-starting capabilities. Bachant and Wosnik [41] studied the helical blade design for a cylindrical and spherical turbine on a lab-scale hydrokinetic turbine for an overlap ratio of 0.5. Marsh et al. [42] suggested that the straight-bladed VAWT could produce more power compared to the helical-bladed VAWT. The study was conducted for a $60^{\circ}$ helical VAWT and tries to prove that the load on the shaft reduces considerably because of the helicity of the blades. The study on a helical-bladed small-scale $(\mathrm{D}=0.5 \mathrm{~m})$ VAWT by Pongduang et al. [43] for different helical blades $\left(120^{\circ}, 130^{\circ}\right.$, and $\left.150^{\circ}\right)$ concludes that the turbine fails to generate 
power at higher helix angles of the blade. However, the study on the helix angle is not comprehensive enough in proving the effect of the same on large-scale turbines.

Although there have been studies on helical-bladed VAWT, the unavailability of analytical research on commercial-scale VAWTs and the effect of change in helix angle on the aerodynamic performance of a VAWT are the motivation behind this work. Researches in helical-bladed VAWT focus most of their aerodynamic study on altering the TSR [28-30] and changing helix angles [36,41-43]. However, those studies are limited to maximum helix angles of $135^{\circ}$ and lab-scale models. A study based on commercial small-scale turbines is essential, as it can help the development and implementation of VAWT in situ. The objective of this work is to understand the effect of varying helix angles $\left(60^{\circ}\right.$ to $\left.120^{\circ}\right)$ on the performance of VAWT under different operating conditions of TSR. The study also tries to compare the performance of these helical turbines to a straight blade VAWT of similar swept area. The primary effects on the aerodynamic performance and the continuity of power generated from the turbines are analysed. The effect of helix angle on the loads on various sections of the blade is also studied in detail.

The purpose of the study is to investigate the effect of helical angles on the aerodynamic performance of a helical VAWT. Straight-bladed VAWT have a ripple effect on the shaft. The ripple nature of the loading comes because of the limited operating zone of a blade during its entire cycle. The cyclic nature of loading on the wind turbine shaft causes critical failures in the structure. Hence, increasing the operating angle of each blade is found to be a possible solution.

\section{Numerical Model}

For this study, blade geometry is generated by helically extruding NACA0015 airfoil with no pitch. The airfoil is helically swept at $60^{\circ}, 90^{\circ}$, and $120^{\circ}$ for obtaining the geometry. A shaft of $200 \mathrm{~mm}$ diameter is considered essential for better prediction of the flow field. At any cross-section of the turbine perpendicular to the axis of rotation, the cut section area of the blade would be the standard airfoil with its centroid lying on the cylindrical surface generated by the rotation of the turbine. Although the manufacturing of these helical blades is difficult using conventional manufacturing processes, contemporary techniques such as additive manufacturing have laid the foundation for manufacturing near-impossible geometries with ease and perfection. The blades have a chord of $210 \mathrm{~mm}$ length and a diameter of $1 \mathrm{~m}$ (leading to the solidity of 0.4 ), and the turbine has a height of $3 \mathrm{~m}$. Since the blade has a more extended azimuth angle of operation, the analysis of the turbine without the central shaft would lead to a wrong prediction of the flow field.

Figure 1a shows the direction of rotation $(\theta)$ of the turbine and the definition of helix angle $(\psi)$. The blue airfoil represents the bottom plane, and the red airfoil represents the top plane of the turbine. Figure $1 \mathrm{~b}-\mathrm{d}$ represents the geometry of $60^{\circ}, 90^{\circ}$, and $120^{\circ}$ helical-bladed VAWT, respectively.

The inlet wind velocity is in the positive y-direction. The wind velocity direction is considered uniformly across all the analysis in this study.

Flow across the turbine is always in the low Mach number range; hence, it is a reasonable assumption to consider the flow to be incompressible. The conservation of mass (continuity) and momentum (Navier-Stokes) equations for a three-dimensional incompressible isothermal flow are solved. The Reynolds number of the flow over the blade ranges from $1.4 \times 10^{5}$ to $3.5 \times 10^{5}$. Therefore, the turbulence is modelled using transitional SST k- $\omega$ model. Rezaeiha et al. [44] have concluded that among all the turbulence models, transition SST k- $\omega$ works better for VAWT simulations. Transition SST k- $\omega$ is used because the flow around VAWT is in a transition regime, and it is difficult to characterise the flow as fully laminar or fully turbulent. The governing equations, i.e., conservation of mass (continuity) and momentum (Navier-Stokes equations) used in the numerical modelling are as follows:

$$
\nabla \cdot \rho \vec{V}=0
$$




$$
\begin{gathered}
\rho \frac{D \vec{V}}{D \mathrm{t}}=-\nabla p+\mu \nabla^{2} \vec{V} \\
\rho\left[\frac{\partial(k)}{\partial t}+\frac{\partial\left(\rho u_{j} k\right)}{\partial x_{j}}\right]=\hat{P}_{k}-\hat{D}_{k}+\frac{\partial}{\partial x_{j}}\left[\left(\mu+\sigma_{k} \mu_{t}\right) \frac{\partial k}{\partial x_{j}}\right] \\
\rho\left[\frac{\partial(\omega)}{\partial t}+\frac{\partial\left(u_{j} \omega\right)}{\partial x_{j}}\right]=P_{\omega}-D_{\omega}+\frac{\partial}{\partial x_{j}}\left[\left(\mu+\sigma_{\omega} \mu_{t}\right) \frac{\partial \omega}{\partial x_{j}}\right]+2\left(1-F_{1}\right) \frac{\rho \sigma_{\omega 2}}{\omega} \frac{\partial k}{\partial x_{j}} \frac{\partial \omega}{\partial x_{j}} \\
\rho\left[\frac{\partial(\gamma)}{\partial t}+\frac{\partial\left(\rho u_{j} \gamma\right)}{\partial x_{j}}\right]=P_{\gamma}-D_{\gamma}+\frac{\partial}{\partial x_{j}}\left[\left(\mu+\frac{\mu_{t}}{\sigma_{f}}\right) \frac{\partial \gamma}{\partial x_{j}}\right] \\
\rho\left[\frac{\partial\left(\hat{R}_{\theta t}\right)}{\partial t}+\frac{\partial\left(u_{j} \hat{R} e_{\theta t}\right)}{\partial x_{j}}\right]=P_{\theta t}+\frac{\partial}{\partial x_{j}}\left[\sigma_{\theta t}\left(\mu+\mu_{t}\right) \frac{\partial \hat{R}_{\theta t}}{\partial x_{j}}\right]
\end{gathered}
$$

where $\rho$ is the density, $V$ is the velocity, $\mu$ is the dynamic viscosity, $p$ is the pressure, $\hat{P}_{k}, P_{\omega}$, $P_{\gamma}, P_{\theta t}$ are the production terms for respective quantities, $\hat{D}_{k}, D_{\omega}, D_{\gamma}$ are destruction terms of the respective quantities.

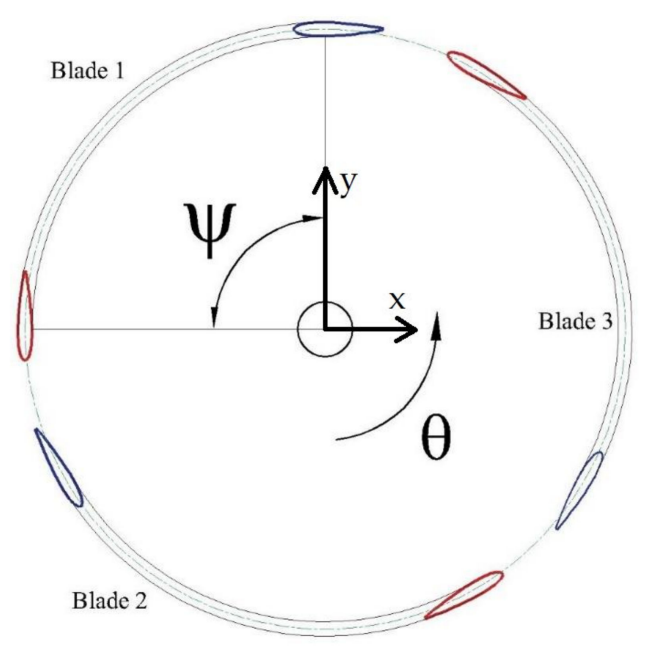

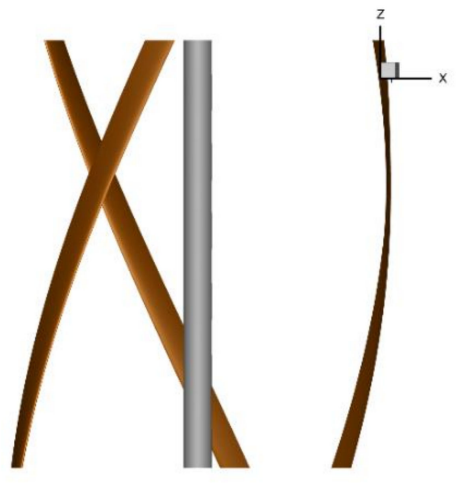

(b)

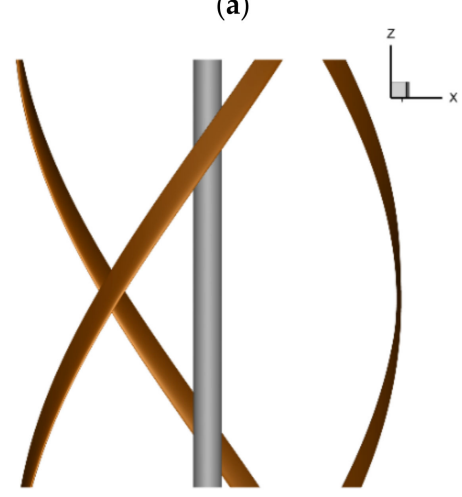

(c)

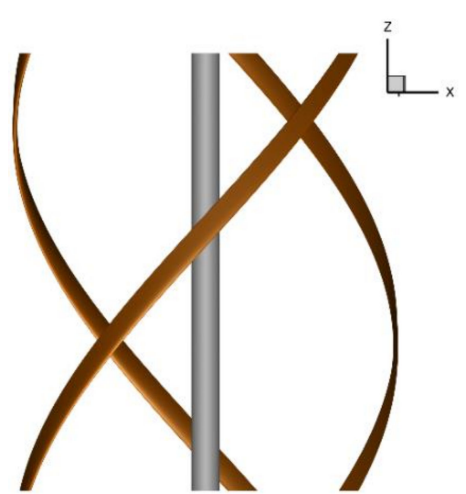

(d)

Figure 1. Top view of (a) Helical blade vertical axis wind turbines (VAWT) with definition of direction of rotation $(\theta)$ and helix angle $(\psi)$; front view of helical blade VAWT (b) $\psi=60^{\circ}$ (c) $\psi=90^{\circ}$ (d) $\psi=120^{\circ}$.

\subsection{Computational Domain and Grid}

The numerical domain is divided into two: a stationary and rotating domain with a mesh interface defined for proper continuity. The rectangular stationary domain shown in Figure 2 has been defined based on the reference value of turbine diameter $\mathrm{D}$, with $15 \mathrm{D}$ length in the upwind and 30D in the downwind measured from the shaft centre. A width 
of 40D was defined to avoid any wall blockage effects. The height of 4.5 times that of the turbine diameter was defined for the domain. The rotating domain with mesh motion was defined to be three times the diameter of the turbine. Mesh in a section plane perpendicular to the turbine axis (z-axis) is shown in Figure 3a. It can be noticed that the rotating domain is given sufficient refinement to capture the flow fields near the blades and shaft. Figure $3 \mathrm{~b}$ shows the boundary layer of $2.5 \times 10^{-5} \mathrm{~m}$, which is defined near the blade walls. The controlled growth of mesh farther away from the blade is ensured by using various control volumes, as shown in Figure 3c. In Figure 3b, the velocity inlet and pressure outlets are shown. The inlet velocity plane is at a distance from the centre of the turbine 15 times that of the diameter of the turbine. The outlet pressure plane is 30 times the diameter from the centre of the turbine at the downstream. The sidewalls and the top and bottom surfaces are defined to be free shear walls.

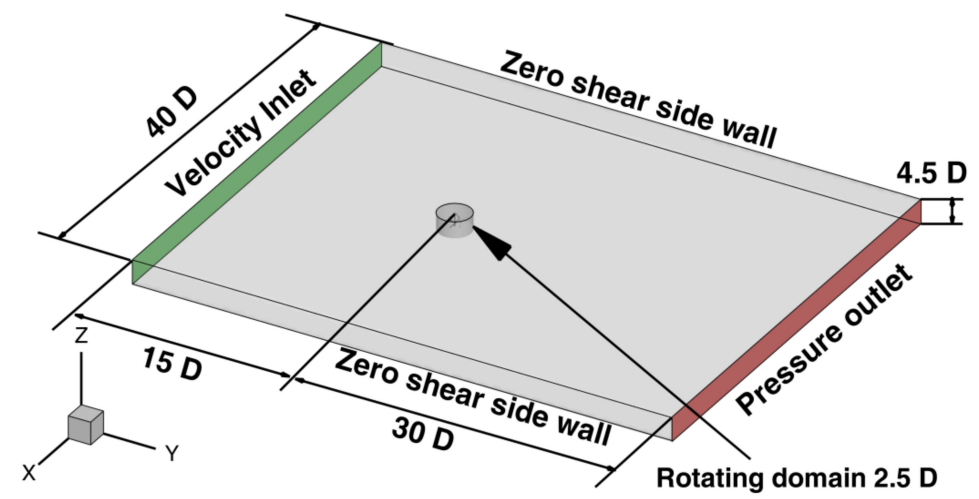

Figure 2. Domain consisting of the stationary cuboidal domain and rotating cylindrical domain.

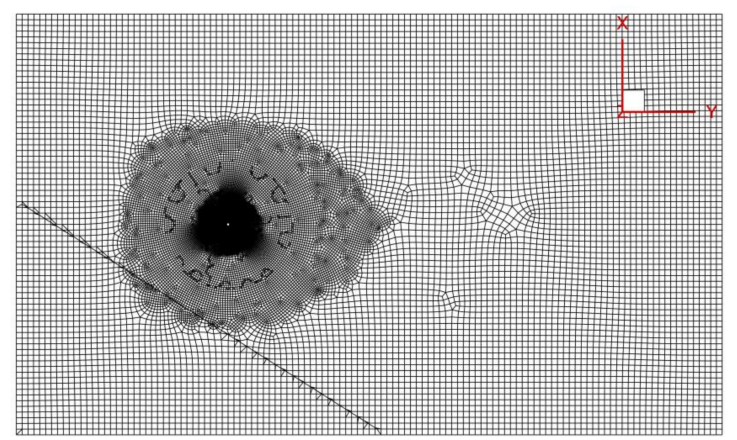

(a)

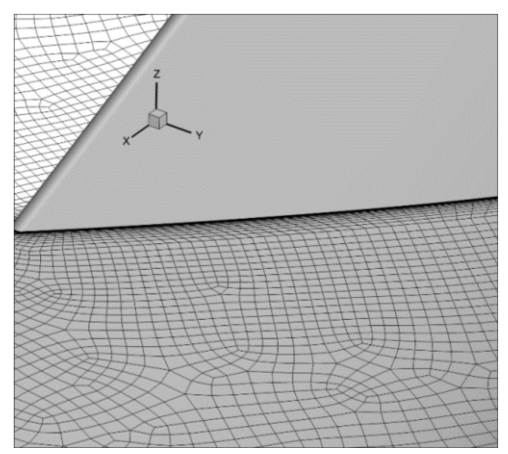

(b)

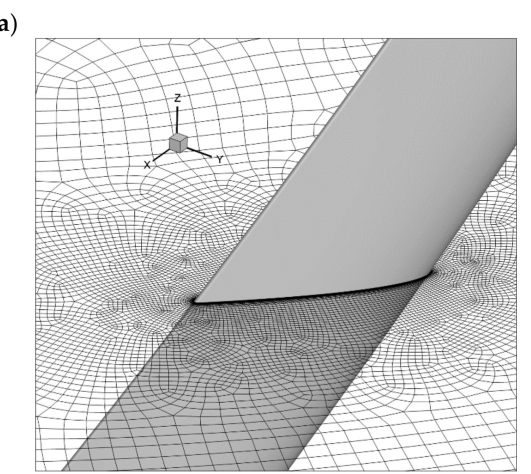

(c)

Figure 3. (a) Sectional view of the domain mesh: (b) mesh near the blade, (c) mesh growth surrounding the blade.

\subsection{Boundary, Initial Conditions, and Computational Procedure}

The rectangular numerical domain considered consists of two side walls and top and bottom planes with zero shear. Inside the rectangular domain, we have a cylindrical 
rotating domain which is interfaced with a sliding mesh interface. The rotating mesh is allowed to rotate about the $z$-axis, and the rotation is defined based on the tip speed ratio at which the simulations are run. It is determined by the formula: $N=\frac{60 \times \mathrm{TSR} \times \mathrm{U}_{\infty}}{2 \times \pi \times \mathrm{R}}$ where $\mathrm{N}$ is the number of revolutions per second, TSR is the tip speed ratio, $\mathrm{U}_{\infty}$ is the free stream velocity $(10 \mathrm{~m} / \mathrm{s})$, and $R$ is the radius of the turbine. It has to be noted that when the tip speed ratio is fixed, then the corresponding rotation for the rotating interface is defined. Hence the rotation of the domain for each simulation is defined depending on the TSR. The blades and shaft surfaces are defined as no slip. The grid near to the top and bottom surfaces of the turbine blades and shaft are refined to compute the tip effects. The flow was initialised from the velocity inlet at $10 \mathrm{~m} / \mathrm{s}$. The rotation is initiated with and run for 10 cycles so that the results have converged. The time steps size is defined based on the rotation angle. Hence for each TSR, the time step size is defined.

The algorithm used for pressure velocity coupling is COUPLED [45]. The convective scheme employed for spatial discretisation is a second-order upwind scheme. The second order in time is used for transient terms. The under relaxation factor for turbulent kinetic energy, specific dissipation rate, intermittency, and momentum thickness Re was set to 0.4 . The turbulent viscosity under the relaxation factor was set to be 0.5. For each time step, 40 iterations were performed such that the residuals for all the variables would converge to an order of $10^{-5}$.

\subsection{Model Validation and Solution Independence}

Grid independence study is conducted to maintain consistency in the solution and reduce the computational effort. Three models of 9 million, 14 million, and 21 million grid points were solved to get solution for 15 cyclic revolutions of the turbine. Figure $4 \mathrm{a}$ represents the moment coefficients of a single blade, which predict similar results for the above-mentioned three grids. Hence, for all the numerical calculations, the 9 million grid mesh was employed. The solutions were found to be in an agreeable range. The time resolution also plays a critical role. The time step was defined based on the azimuth angle of rotation of the turbine. Three different resolutions $\left(2^{\circ}, 1^{\circ}\right.$, and $\left.0.5^{\circ}\right)$ were tried and tested to make sure the results predicted are reasonable. From Figure $4 \mathrm{~b}$, it is evident that all three-time step definitions are predicting similar solutions with significantly fewer errors.

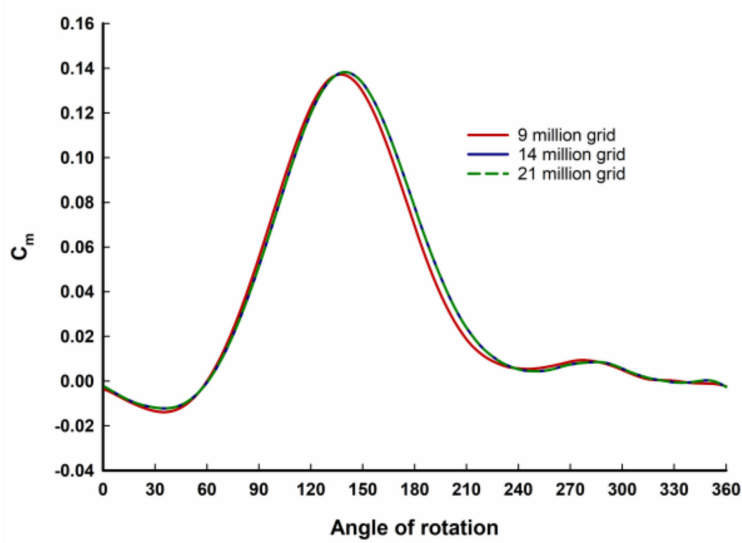

(a)

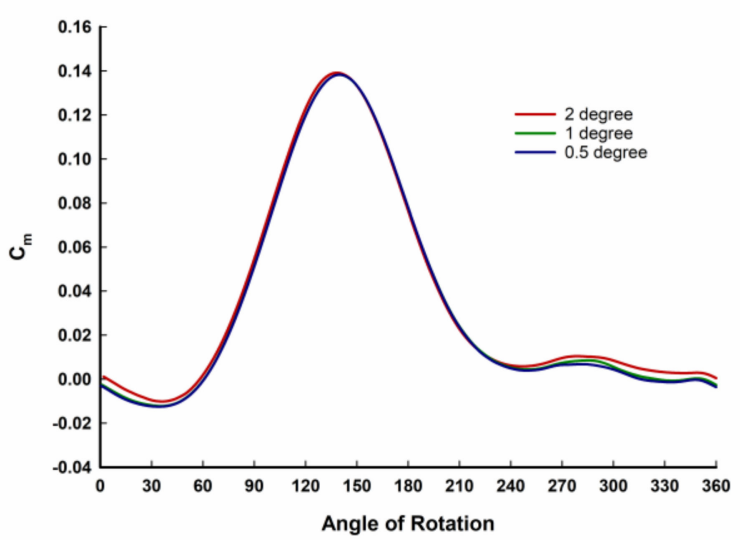

(b)

Figure 4. Moment coefficient $(\mathrm{Cm})$ plot for a single blade indicating (a) grid independence, (b) time step independence.

Figure 5 illustrates the cyclic convergence for a tip speed ratio of $2.3,60^{\circ}$ helical-bladed VAWT case. The solution has achieved a cyclic nature by the end of the 10th cycle itself, which indicates that the solution has reached convergence. All the simulations were run for 14 rotation cycles of the turbine to remove any non-cyclic behaviour in the solution. 


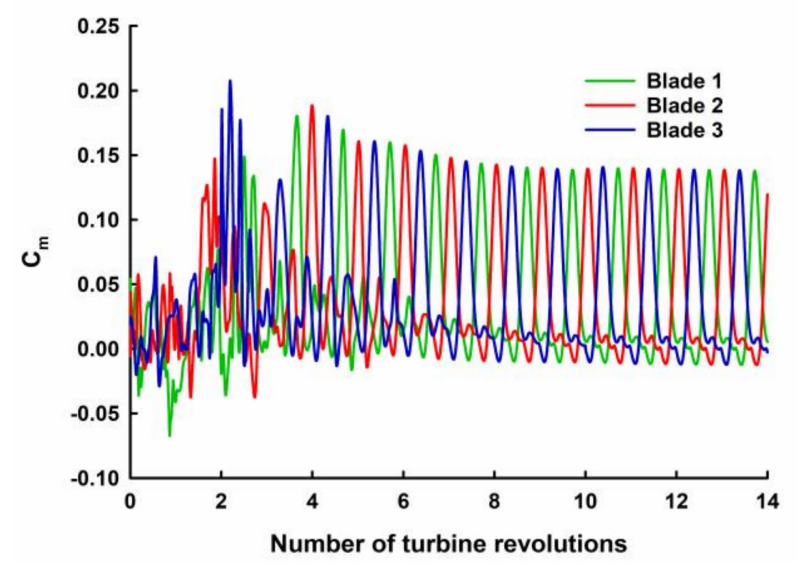

Figure 5. Cyclic convergence of solution for a $60^{\circ}$ helical blade VAWT running at $\lambda=2.3$.

To validate the numerical model used in this study, McLaren's experimental data [46] were used. In the experimental study, a $420 \mathrm{~mm}$ chord three-bladed straight VAWT was studied and tested in the wind tunnel. The numerical model with similar dimensions was simulated. The numerical results of power coefficient deviated a maximum of $8 \%$ with respect to experimental data, as shown in Figure 6. This gave considerable confidence to the numerical model settings that are used in this study. Moreover, the experimental uncertainity reported by McLaren was $\pm 14.5 \%$. It should also be noted that in the present work, the helical turbine studied has same dimensions of McLaren's straight VAWT.

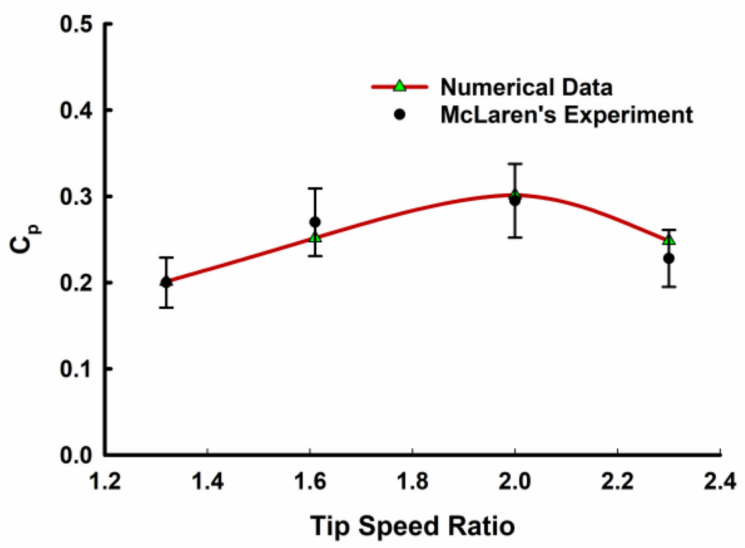

Figure 6. Validation of numerical results to McLarens experimental results.

\section{Results}

\subsection{Effect of Helix Angle on the Performance of VAWT}

The performance of vertical axis wind turbines for various helix angles operating over a range of tip speed ratios (TSRs) is plotted in Figure 7. The performance of straight blade VAWT and different helical VAWTs are compared in this figure. It is seen that the straight blade VAWT showcases its best performance in a lower TSR range (2.7-3), but when a helix angle is introduced, the performance curve shows a shift in the peak, maintaining a similar overall trend of the curve. For a helix angle of $60^{\circ}$, the performance is maximum at a TSR of 3.3, showcasing better performance compared to all other helical and straight blade turbines. For helix angles $90^{\circ}$ and $120^{\circ}$, the performance is comparable with a straight blade turbine, but the performance is peaking at a higher TSR. It is evident that the best performance is projected for the $60^{\circ}$ helical blade turbine, but the slope in the curve is too large for a small variation of TSR. For a $120^{\circ}$ helical blade turbine, the performance curve looks far inferior to any other turbines that are considered. 


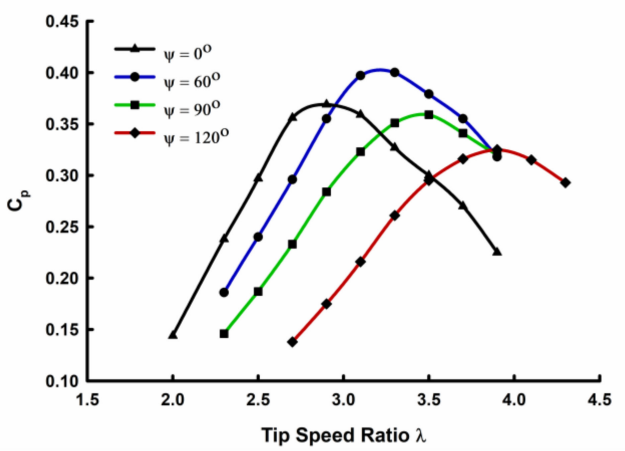

Figure 7. Coefficient of performance of VAWT for various helix angles.

The moment coefficient $\left(\mathrm{C}_{\mathrm{m}}\right)$ of a single blade is plotted against the azimuthal angle of rotation in Figure $8 \mathrm{a}-\mathrm{c}$ for helix angles of $60^{\circ}, 90^{\circ}$, and $120^{\circ}$, respectively. The $C_{\mathrm{m}}$ curve at different TSRs $(\lambda)$ for different helix angles are plotted for each helical turbine. It should be noted that the coefficient of performance is the product of TSR and average $C_{m}$. Irrespective of helix angle, the peak performance of each blade keeps shifting to the higher azimuth angle as the TSR increases. As the helix angle increases, the shift in occurence in peak $C_{m}$ is significant. For the $60^{\circ}$ helix angle, the peak of the $C_{m}$ curve shifts in the range of $135^{\circ}$ to $180^{\circ}$ of the azimuth angle for a TSR range of 2.3 to 3.5 , whereas for the $120^{\circ}$ helical blade VAWT, the peak of the $C_{m}$ curve shifts from $45^{\circ}$ to $135^{\circ}$ of the azimuth angle for the same TSR range. This can be attributed to the reason that the increase in the helix angle would lead to exposure of the blade to the incoming wind for a larger azimuth angle. It is also interesting to see the secondary peaks, which may be due to the significant reduction in wake interaction when the TSR goes higher. The slope of the curve also increases as the helix angle decreases. This will have an adverse effect on the load that gets transmitted on to the shaft. A cross-comparison of helix angles (Figure 9a-d) at $\lambda=2.3,2.7,3.1,3.5$, and 3.9 shows that the straight blade VAWT had the highest peak while the helical VAWT had the lowest peak. It is interesting to look at the curve slope for each helix angle blade. The curve is steep for lower helix angles. To further investigate the effect of the slope of the curve on the performance, a normalised cumulative $C_{m}$ was plotted.
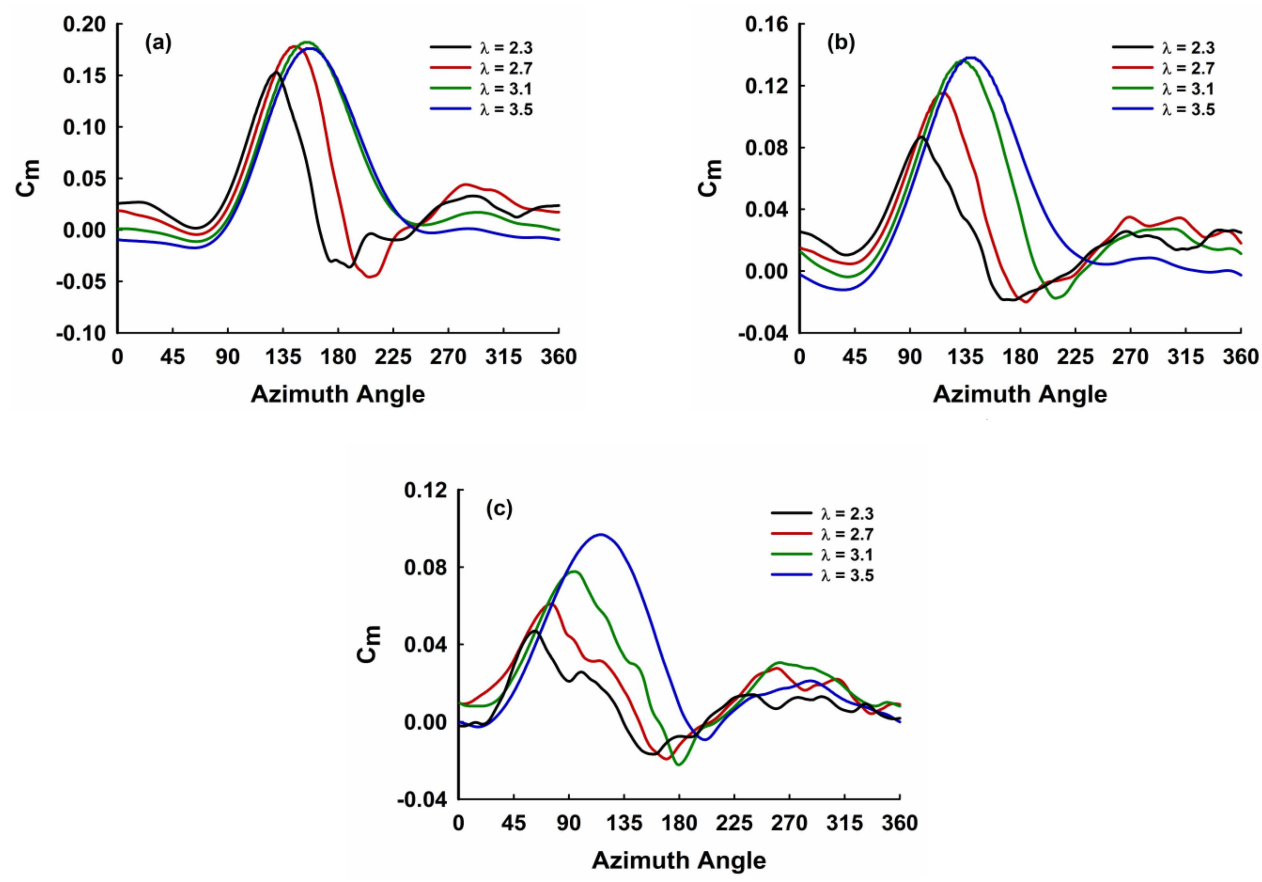

Figure 8. Moment coefficient for various tip speed ratios (TSRs), (a) helix angle $60^{\circ}$, (b) helix angle $90^{\circ}$, (c) helix angle $120^{\circ}$. 

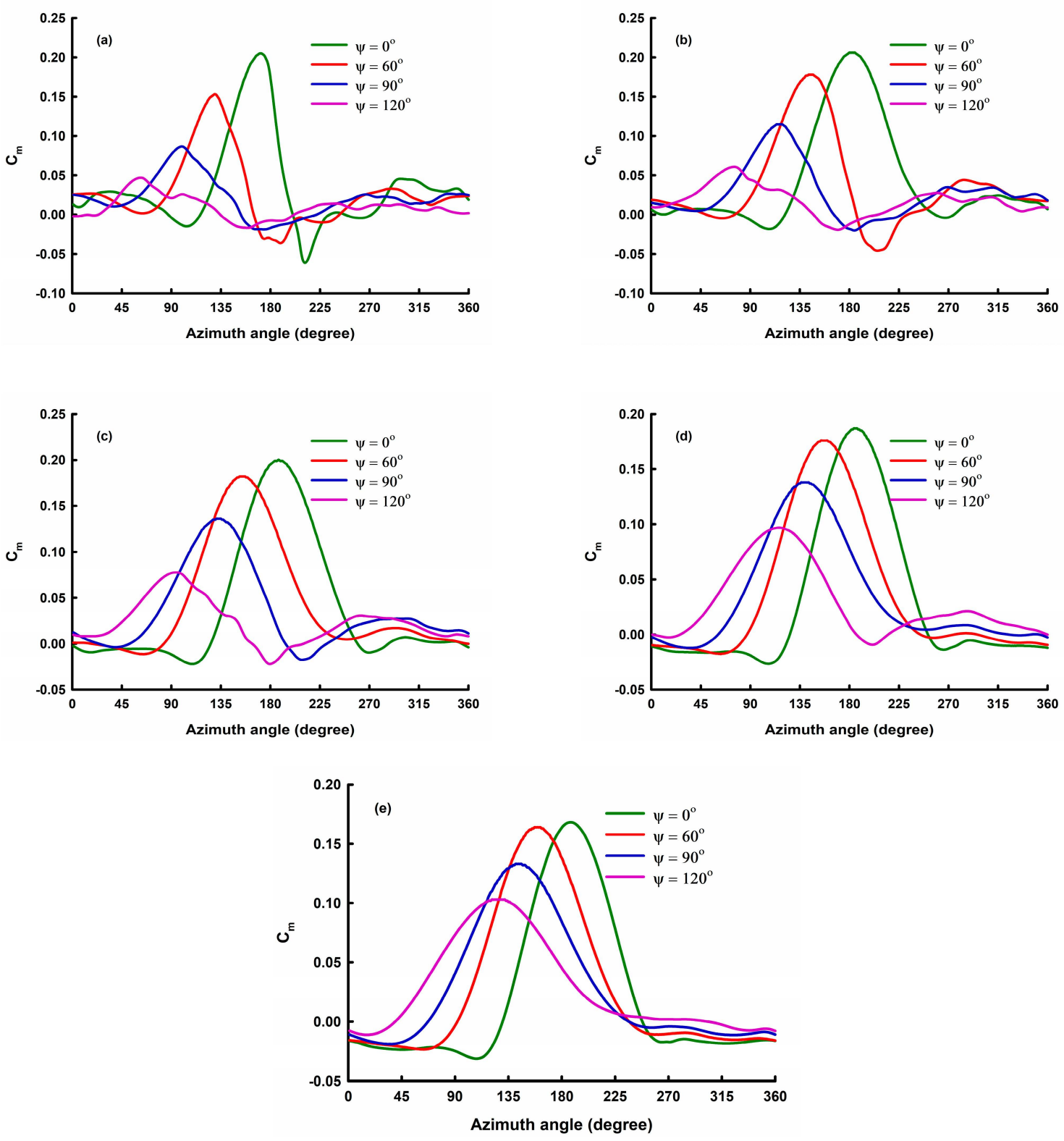

Figure 9. $C_{m}$ curve for the first blade of straight $60^{\circ}, 90^{\circ}$, and $120^{\circ}$ helix angles of blades compared at (a) $\lambda=2.3,(\mathbf{b}) \lambda=2.7$, (c) $\lambda=3.1$, (d) $\lambda=3.5$, and (e) $\lambda=3.9$.

In Figure 10, the normalised curve was plotted, which gave a better understanding of the nature of energy generated. The trend of power generated in each of these cases was similar except that the slope of the curve was decreasing as the helix angle increased. This is attributed to the broader range of azimuth angle in which the power gets generated. It must be noted that the normalised curve also gave an understanding of how the trend behaved when the tip speed ratio was changed at different helix angles. When we compare the slope of cumulative $C_{m}$ for different helix angles performing at the same TSR, the slope variation can be understood. The straight blade can be seen going to negative and then going above 1 . This is mainly because of the limited azimuth angle of positive operation. In contrast, the curve for $120^{\circ}$ has a less steep curve, and most of the portion of the curve is positive. The negative slope in the cases of $90^{\circ}$ and $120^{\circ}$ are impressive because they represent the secondary wake interactions. It would be interesting to see the cumulative effect of all three blades on the turbine power generation curve. Hence, the cumulative $C_{m}$ curve as plotted in Figure 11. 

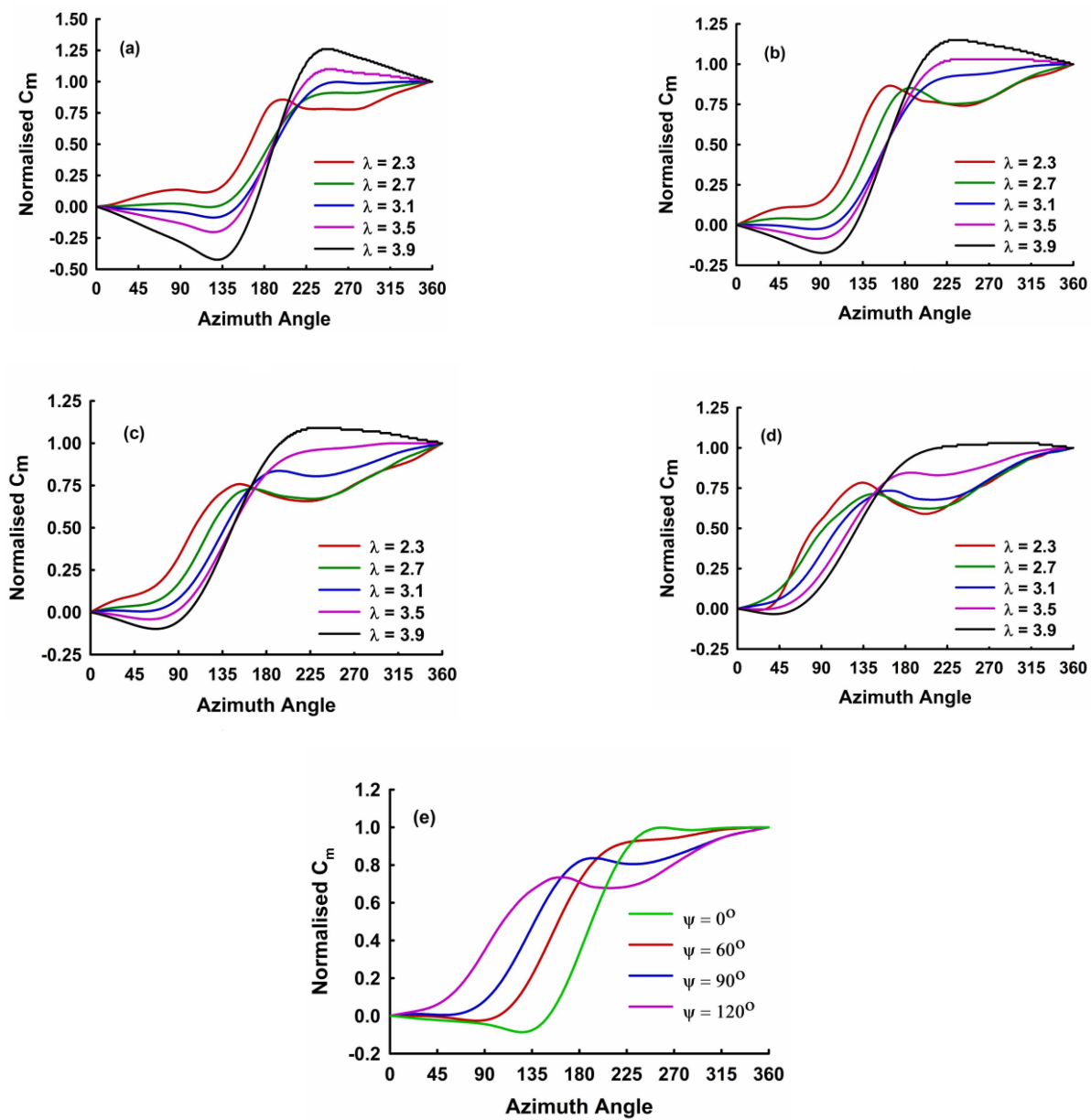

Figure 10. Normalised cumulative $C_{m}$ Curve of blade 1 at different $\lambda$ for (a) $\psi=0^{\circ},(\mathbf{b}) \psi=60^{\circ}$ (c) $\psi=90^{\circ}$, (d) $\psi=120^{\circ}$, (e) Normalised curve of all helical-bladed turbines compared against straight blade turbine at a TSR of 3.1 .
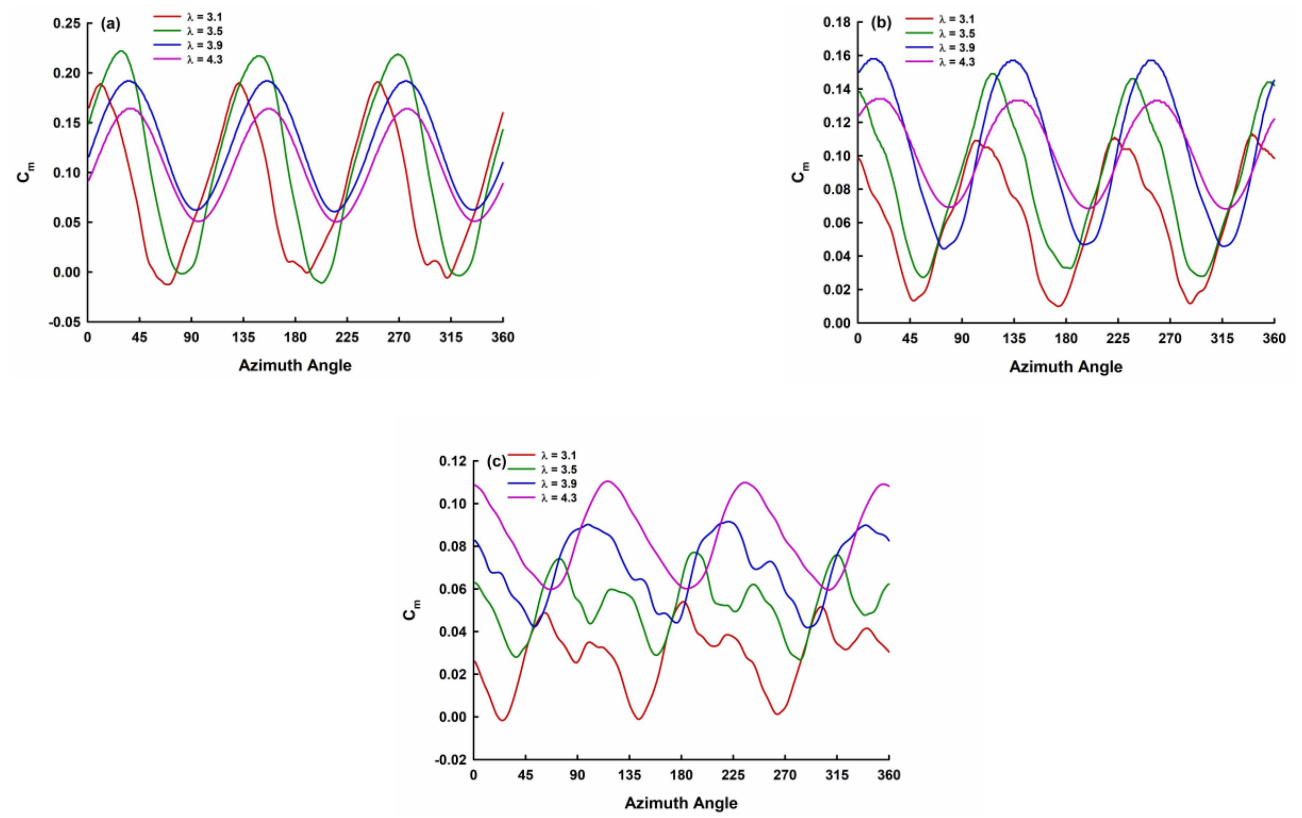

Figure 11. Cumulative $C_{m}$ plot for helical turbine having (a) $\psi=60^{\circ},(\mathbf{b}) \psi=90^{\circ}$, and (c) $\psi=120^{\circ}$. 
The cumulative $C_{m}$ effect of each turbine was plotted for different tip speed ratios in Figure 12. It was found that the $C_{m}$ curve had a maximum point and a minimum point. These fluctuations in maximum and minimum are large for lower TSRs for all the types of turbines. For $60^{\circ}$ and $90^{\circ}$ helical-bladed turbines, it is noticed that the fluctuations minimise as the TSR increases. It is seen that for lower TSRs, the $C_{m}$ curve has a lot of instability for a $120^{\circ}$ helical blade when operating at a lower TSR. The possibility of wake interaction is higher, since the blades have higher overlap in the circumference of the turbine.
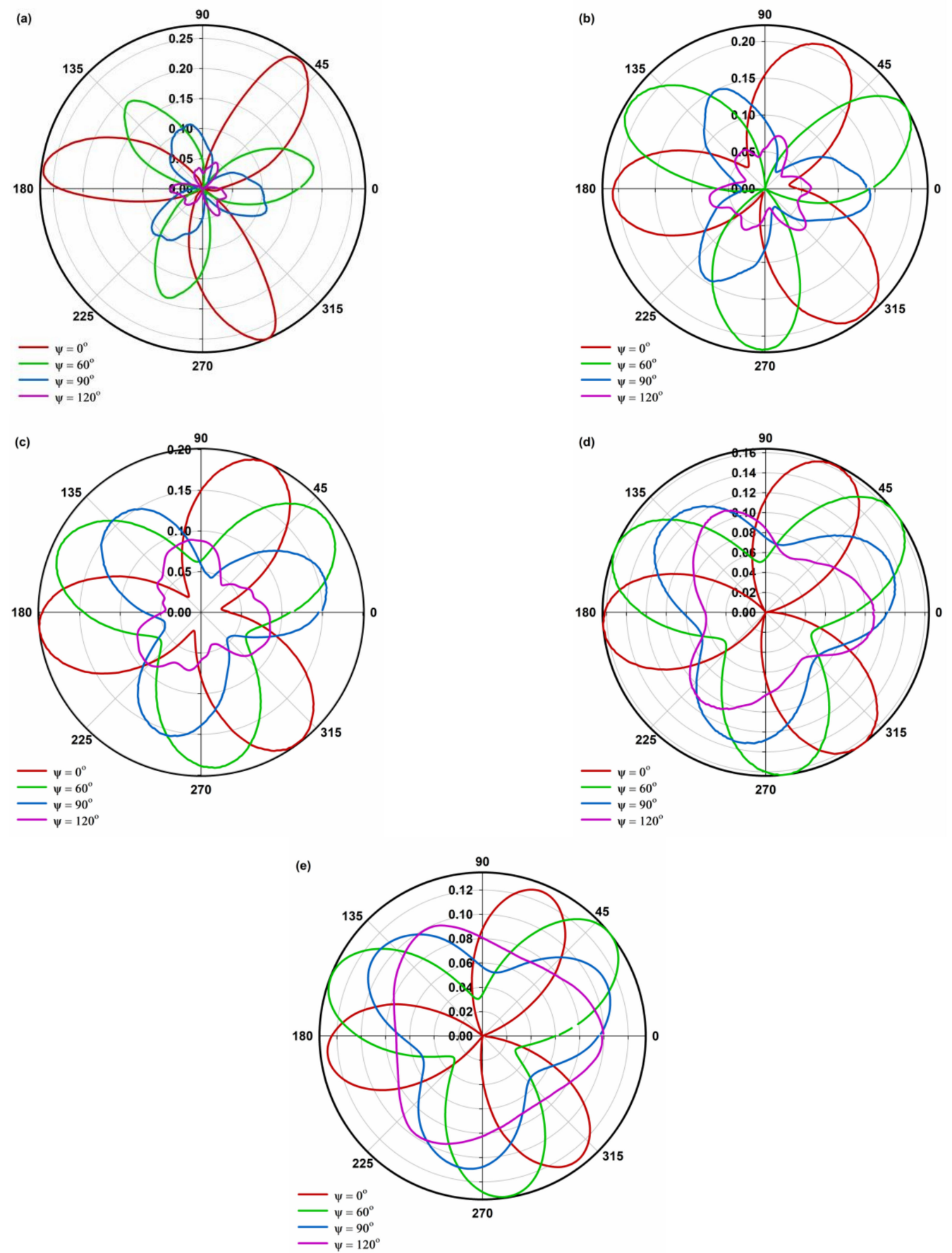

Figure 12. Cumulative polar plot of $C_{m}$ comparing different helix angles and a straight blade at (a) TSR $=2.3,(\mathbf{b})$ TSR $=2.5$, (c) $\mathrm{TSR}=3.1,(\mathbf{d}) \mathrm{TSR}=3.5,(\mathbf{e}) \mathrm{TSR}=3.9$. 
The plot is trying to compare the performance of different helical-bladed VAWTs operating in the same TSR. However, the trends look quite similar for low and medium TSR; at higher TSR, the turbine having 120 helical-bladed VAWT had a lesser deviation from the mean performance curve over the cycle.

In Figure 13, the definition of quartile and the contribution of each turbine quartile to the total moment generated is plotted to compare different helical turbines operating at TSR 2.3 to 3.9. The contribution is calculated as the difference between the cumulative sum of normalised $C_{m}$ at the end and the start of each quartile. For several cases, it is seen that the percentage of contribution crosses $100 \%$. This is mainly due to transitions from negative values of $C_{m}$ at the beginning of the quartile to a more considerable positive value of $C_{m}$ by the end of the quartile. It is also noticed that for such cases, the percentage contribution of the blades will go into negative values in other quartiles as the sum total has to be $100 \%$.
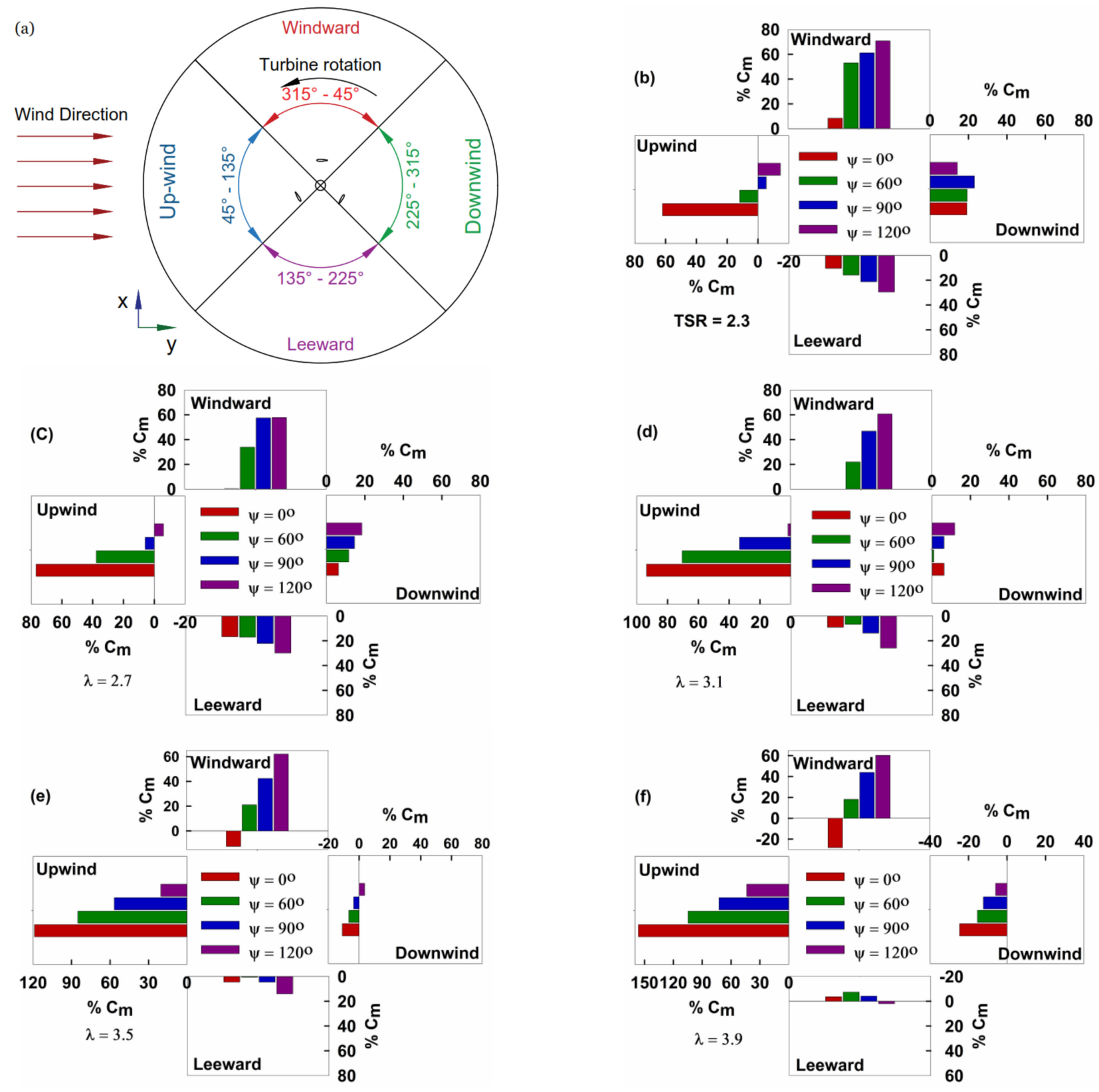

Figure 13. (a) Definition of quartile and percentage contribution by a blade in each quartile for all helical blades and straight blades at (b) TSR $=2.3,(\mathbf{c}) \mathrm{TSR}=2.7,(\mathbf{d}) \mathrm{TSR}=3.1,(\mathbf{e}) \mathrm{TSR}=3.5$, and $(\mathbf{f}) \mathrm{TSR}=3.9$. 
It is interesting to note that the percentage contribution in the downwind side is positive for lower TSR. However, as the TSR increases, the downwind quartile contribution tends to go to negative contributions. This can be attributed to the secondary wake interaction at the downstream of the flow at a lower TSR. The leeward side of the flow also follows a similar trend and results in interactions at a lower TSR. At a higher TSR, the difference in the contribution of each quartile becomes large for straight-bladed VAWT, whereas the cumulative contribution of $120^{\circ}$ helical blade VAWT shows positive contribution in all the quartiles (add percentage difference in the quartiles between 60 and 120). If we compare the contributions of each quartile for TSR $=2.3$, it is observed that almost all quartiles contribute positively except for $90^{\circ}$ and $120^{\circ}$ helical VAWT. Straight blade VAWT produce more than $100 \%$ in the upwind quartile for TSR > 3.1. This explains the sudden increase in the slope of the normalised cumulative $C_{m}$ curve. It can be concluded that when the helix angle is introduced, the nature of the power generated over the entire rotation of the turbine changes its characteristics.

The ripple effect on the turbine shaft is understood with the help of standard deviation of $C_{p}$ plotted for different turbines operating at different TSRs. Figure 14 represents the standard deviation of $C_{p}$ of different types of helical-bladed turbines and straight-bladed turbines. The standard deviation reduces as the helix angle increases. The maximum deviation is for straight-bladed VAWT running at 2.3 TSR. For TSR 2.7, the standard deviation of $60^{\circ}$ helical-bladed VAWT overshot the straight-bladed VAWT. The standard deviation of $C_{p}$ is much less for $120^{\circ}$ helical-bladed VAWT than any other configuration of blades. The standard deviation is highest for the straight-bladed VAWT. A straight blade shows the maximum variation, and it is approximately $85 \%$ higher than the $120^{\circ}$ helical-bladed VAWT. The maximum standard deviation for a turbine is away from the peak performance of that turbine.

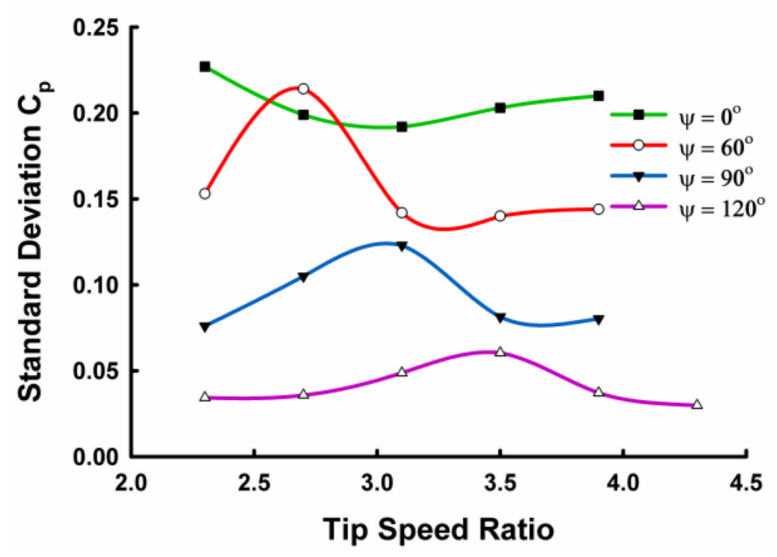

Figure 14. The standard deviation of $\mathrm{C}_{\mathrm{p}}$ of different helical-bladed and straight-bladed VAWT operating at different TSR.

\subsection{Effect of Helix Angle on Turbine Blade Loads}

To understand the power generation distributed across different quartiles of a turbine, it is essential to know how various parts of a helical blade generate the $C_{p}$. The blades were divided into three sections of equal height. The sections are named as the leading, mid, and trailing section, and they are represented in Figure 15 in red, green, and blue colours respectively. The leading section enters any quartile first followed by the mid-section and trailing section while the turbine is in rotation. A sectional analysis is essential in this case the blade interacts with the flow continuously across different quadrants. 


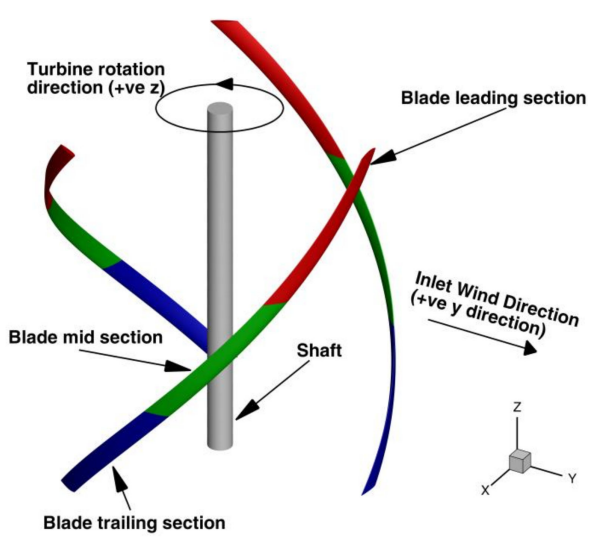

Figure 15. Sections of blades of a helical vertical axis wind turbine.

The $C_{m}$ contribution of each blade segment was observed over a rotation of $360^{\circ}$. The leading section, mid-section, and trailing section $C_{m}$ are plotted in red, green, and blue colours, respectively in Figure 16. Figure 16a-c represents the contribution curves of $60^{\circ}, 90^{\circ}$, and $120^{\circ}$ respectively. The cumulative $C_{m}$ is also plotted in the same plot with a different scale of $y$-axis in black colour. It is interesting to note that the mid-section of the blade contributes marginally more than the other two sections in all helical-bladed VAWT; for $90^{\circ}$ and $60^{\circ}$ helical-bladed VAWT, the trailing section contributes more to the moment coefficient when compared to the leading section. On the contrary, in $120^{\circ}$ helical-bladed VAWT, the leading segment contributes more than the trailing segment when operated in the same TSR of 3.1. It can be noticed from the figure that all sections of blades do not contribute uniformly to the moment coefficient. Drop lines are also plotted in Figure 16 to highlight important azimuth angles. Corresponding to those azimuth angles, the z-vorticity contours are plotted in Figures 17-19. These points are of importance as they represent the peaks of individual segments of the same blade.

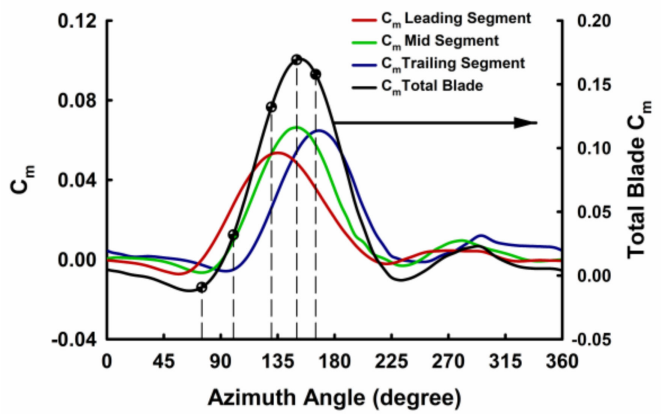

(a)

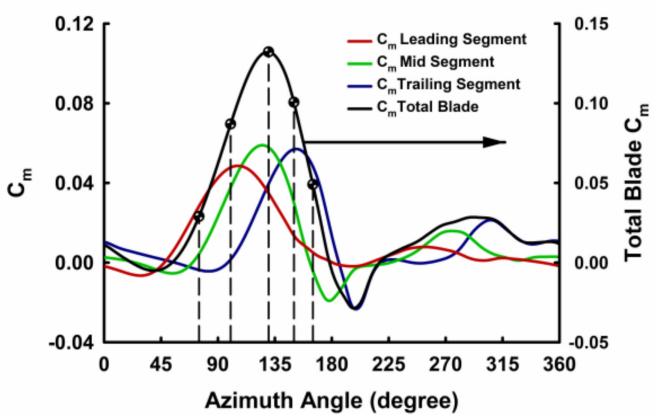

(b)

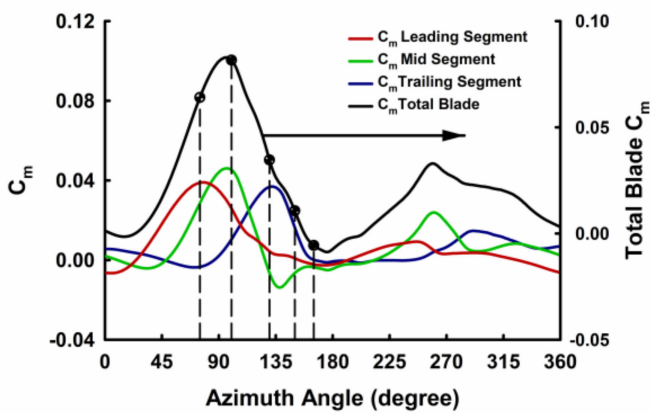

(c)

Figure 16. Section-wise $C_{m}$ contribution of each segment of a single blade of (a) $\varphi=60^{\circ},(\mathbf{b}) \varphi=90^{\circ}$, and (c) $\varphi=120^{\circ}$ helical-bladed VAWT operating at a TSR of 3.1. 


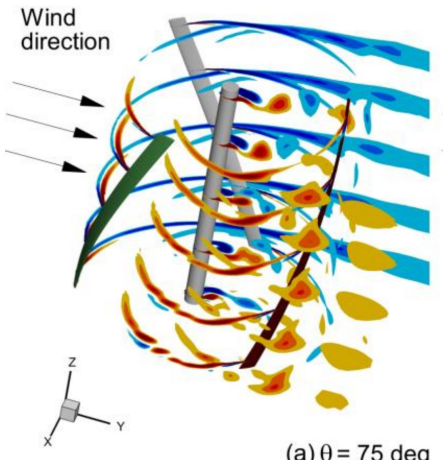

(a) $\theta=75 \mathrm{deg}$

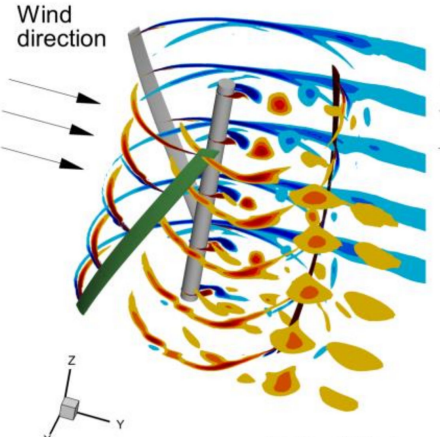

(b) $\theta=100 \mathrm{deg}$

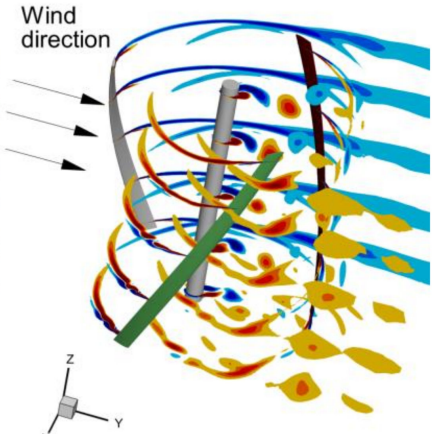

(c) $\theta=130 \mathrm{deg}$

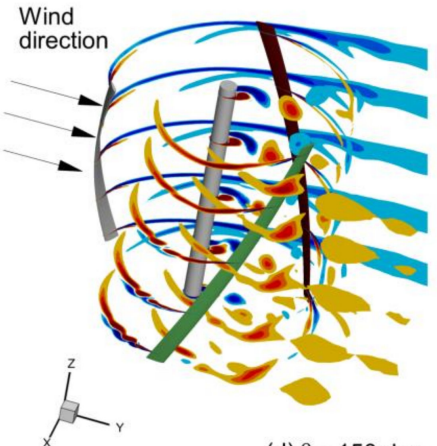

(d) $\theta=150 \mathrm{deg}$

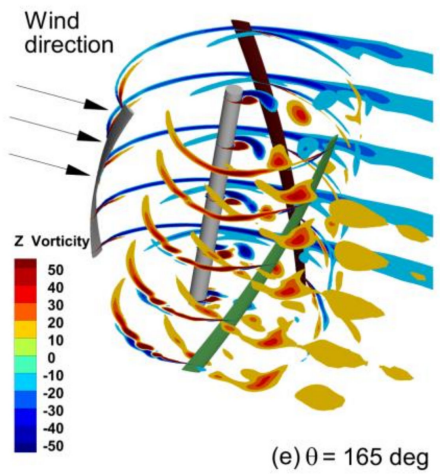

Figure 17. Z-vortex magnitude for $60^{\circ}$ helical VAWT at $\mathrm{z}$ planes at heights of $-1.45 \mathrm{~m},-0.75 \mathrm{~m}, 0 \mathrm{~m}, 0.75 \mathrm{~m}$, and $1.45 \mathrm{~m}$ at azimuth angles of rotation of (a) $75^{\circ}$, (b) $100^{\circ}$, (c) $135^{\circ}$, (d) $150^{\circ}$, and (e) $165^{\circ}$.

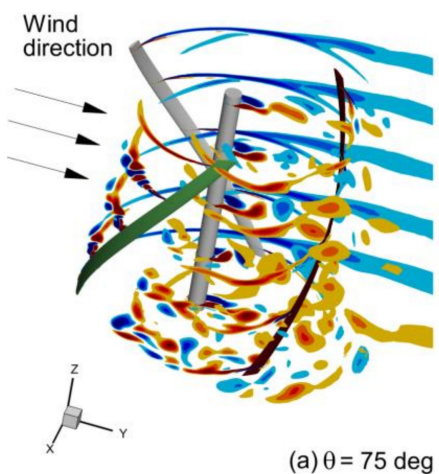

(a) $\theta=75 \mathrm{deg}$

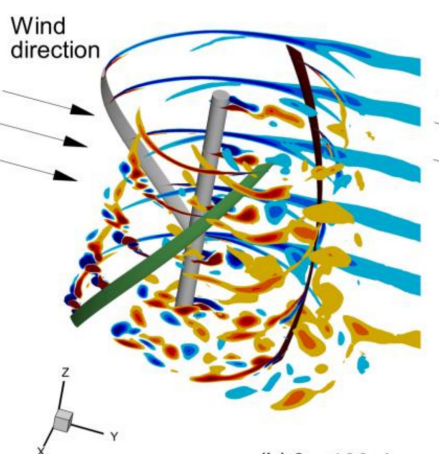

(b) $\theta=100 \mathrm{deg}$

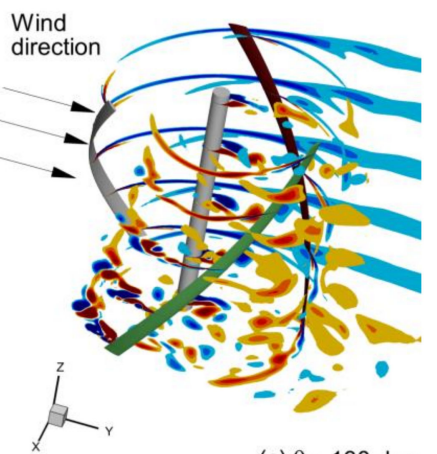

(c) $\theta=130 \mathrm{deg}$
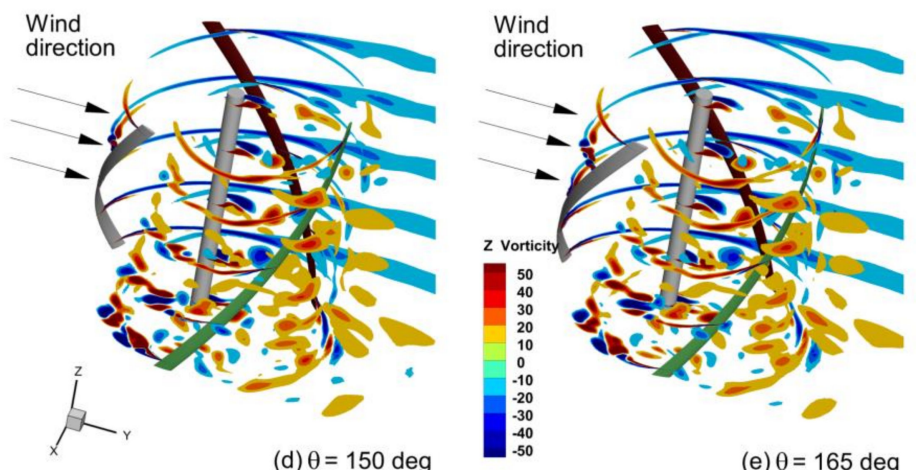

(e) $\theta=165 \mathrm{deg}$

Figure 18. Z-vortex magnitude for $90^{\circ}$ helical VAWT at $\mathrm{z}$ planes at heights of $-1.45 \mathrm{~m},-0.75 \mathrm{~m}, 0 \mathrm{~m}, 0.75 \mathrm{~m}$, and $1.45 \mathrm{~m}$ at azimuth angles of rotation of (a) $75^{\circ}$, (b) $100^{\circ}$, (c) $135^{\circ}$, (d) $150^{\circ}$, and (e) $165^{\circ}$. 


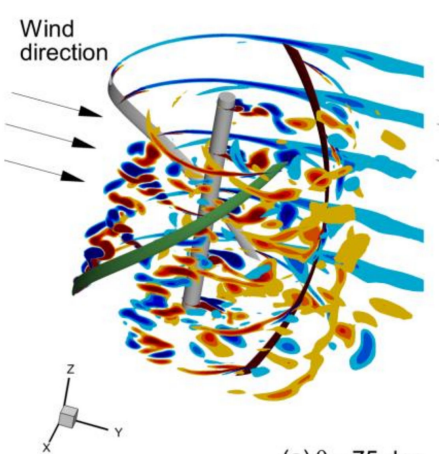

(a) $\theta=75 \mathrm{deg}$

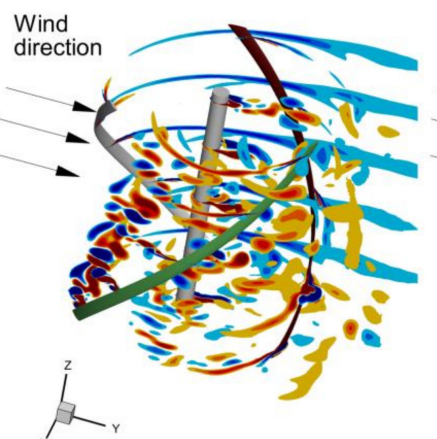

(b) $\theta=100 \mathrm{deg}$

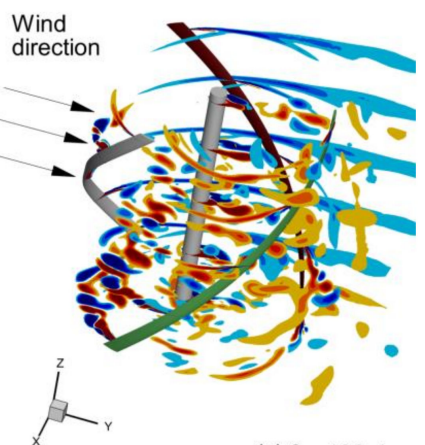

(c) $\theta=130 \mathrm{deg}$

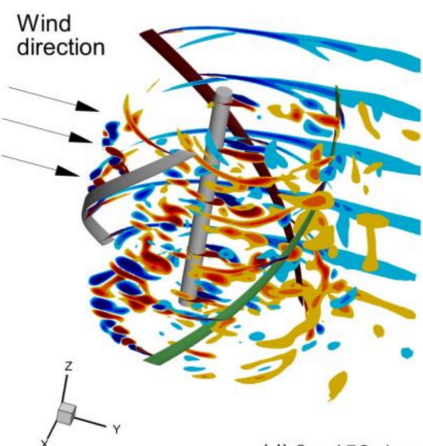

(d) $\theta=150 \mathrm{deg}$

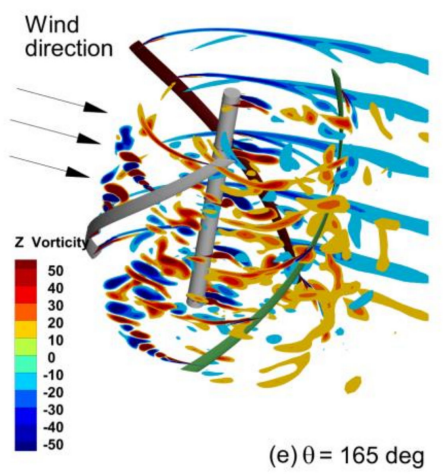

Figure 19. Z-vortex magnitude for $120^{\circ}$ helical VAWT at z planes at heights of $-1.45 \mathrm{~m},-0.75 \mathrm{~m}, 0 \mathrm{~m}, 0.75 \mathrm{~m}$, and $1.45 \mathrm{~m}$ at azimuth angles of rotation of (a) $75^{\circ}$, (b) $100^{\circ}$, (c) $135^{\circ}$, (d) $150^{\circ}$, and (e) $165^{\circ}$.

Figures 17-19 represent the z-vorticity contours of $60^{\circ}$ (Figure 17), $90^{\circ}$ (Figure 18), and $120^{\circ}$ (Figure 19) helical blade VAWT at operating condition of TSR 3.1. It is essential to analyse the flow at different heights of the turbine as the cross-section of the turbine varies along the height. Hence, the flow at different heights $(-1.45 \mathrm{~m},-0.75 \mathrm{~m}, 0 \mathrm{~m}, 0.75 \mathrm{~m}$, and $1.45 \mathrm{~m}$ ) are analysed using these plots. Figure 16 represent the $C_{m}$ curve of blade 1 , which is represented in grey colour for Figures 17-19. Blade 2 and Blade 3 are represented by brown and green colour, respectively. At azimuth angles of $75^{\circ}, 100^{\circ}, 130^{\circ}, 150^{\circ}$, and $165^{\circ}$, the contours are plotted. The vorticity levels are displayed to see the results of magnitude range of -50 to $50 \mathrm{~s}^{-1}$. It is observed for a $60^{\circ}$ helical blade VAWT, at $\theta=130^{\circ}$, that the leading edge of the leading segment of the blade starts forming vortices, and this explains the peak for the leading segment of the blade observed in Figure 16. It is also observed that the leading edge of blade 1 does not shed the vortices in any of the shown frames. The mid segment and trailing segment of Blade 1 start developing leading edge vortices at $\theta=150^{\circ}$ and $\theta=165^{\circ}$, respectively, and the same can be observed in Figure 17d,e. Whereas it can be seen that the leading segment of Blade 3 sheds the vortices in Figure 17a, and in Figure 17e, the flow separation from Blade 3 can also be noticed. The separated flow from Blade 2 can be seen in Figure 17a-e interacting with Blade 3, thereby creating the secondary peaks in the $C_{m}$ plot. The vortex shed by the shaft is also visible in the figure, and it combines with the flow shed by Blade 2 in the downstream.

The z-vorticity plots of $90^{\circ}$ helical-bladed VAWT are plotted in Figure 18. At $\theta=75^{\circ}$, the leading edge vortices can be noticed on the leading segment of Blade 1 . This phenomenon happens to the trailing segment at a much higher azimuth angle when compared to a $60^{\circ}$ helical-bladed turbine. The leading segment makes a peak at around $\theta=100^{\circ}$ and the interactions can be seen from Figure $18 \mathrm{~b}$ that the entire leading segment has entered the flow. The mid segment and trailing segment of the blade starts generating leading edge vortices at $\theta=150^{\circ}$ and $\theta=160^{\circ}$ of the azimuth angle. Meanwhile, flow separation in the leading segment of the blade is noticed in Figure 18d,e. Blade 2 can be seen interacting with the vortex shed by Blade 1 at $\theta=130^{\circ}$. The flow separations from Blade 3 can be seen from $\theta=130^{\circ}$ (Figure 18c), which goes on to shed vortices for the trailing segment at 
$\theta=165^{\circ}$ (Figure 18e). The secondary interactions can be noticed by observing Blade 3 . It is seen interacting with the vortices shed by Blade 2 as early as $\theta=100^{\circ}$. The secondary interactions happen for a longer azimuth angle of rotation, and hence, a wider secondary peak is observed for the $90^{\circ}$ helical-bladed VAWT.

For a $120^{\circ}$ helical-bladed VAWT, it can be seen that the leading segment has already entered the flow at $\theta=75^{\circ}$. The leading segment of the blade can be seen interacting with the vortices of Blade 3 at $\theta=130^{\circ}$ and starts shedding the vortices from the blade surface. The vortex shed by Blade 2 interacts with Blade 3 , and it can be seen that the flow separation occurs at much earlier azimuth angles.

It can be seen from Figures 17-19 that the flow interactions are higher for the $120^{\circ}$ helical blade when compared with other turbines. Blade 3 is coming under the secondary wake interactions produced by Blade 2 at different sections of the blade. This explains the secondary peak observed in the $C_{m}$ plot. It would be interesting to see the flow characteristics for various tip speed ratios. To study the same, a $90^{\circ}$ helical-bladed VAWT's performance was observed. In Figure 20, a $90^{\circ}$ helical-bladed VAWT was observed for the performance of different sections of the blade. As already observed from Figure 7, the performance of the $90^{\circ}$ helical-bladed VAWT improves from TSR 2.7 to 3.1 .

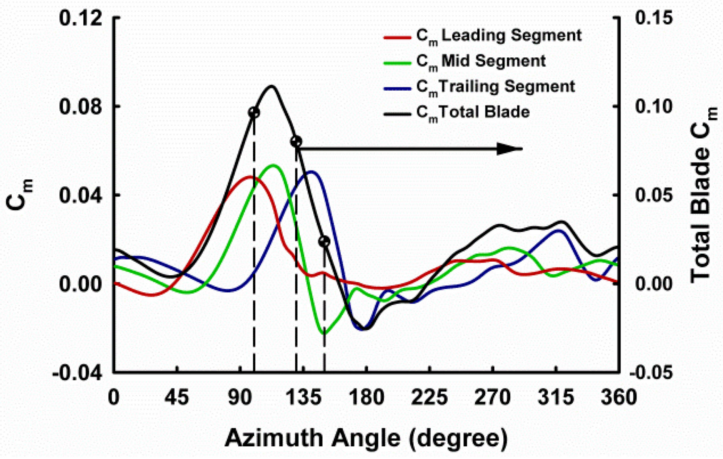

(a)

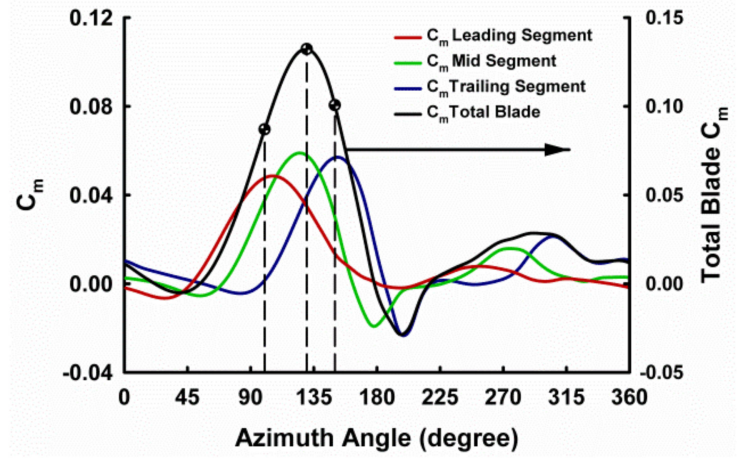

(b)

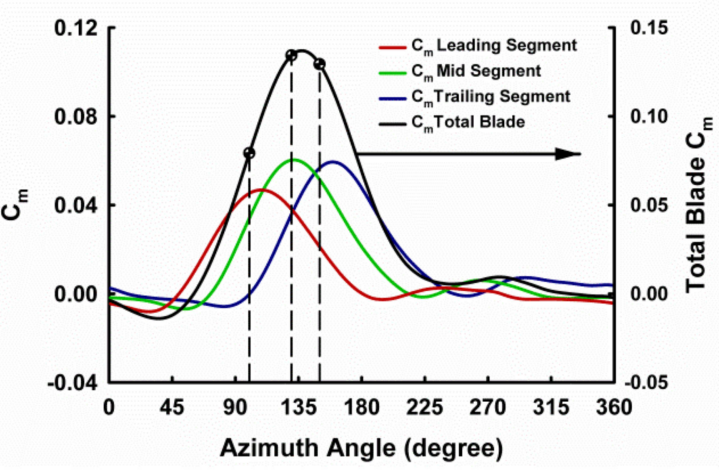

(c)

Figure 20. Section-wise $C_{m}$ contribution of different segments of a single blade of a $90^{\circ}$ helical VAWT operating at TSRs of (a) 2.7 , (b) 3.1 , and (c) 3.5 .

To have a better understanding of how the blade sections behave with a change in TSR, a $90^{\circ}$ helical-bladed VAWT is studied for sectional contributions. From Figure 17, it can be noted that the leading section is contributing less when compared to other sections of the same blade. For a lower TSR, all three sections of the blade contribute an almost equal amount to the cumulative performance of the blade. At TSRs of 2.7 and 3.1, the secondary interactions are higher and seem to contribute positively toward the generation of power. At a lower TSR, the secondary peak is generated. Drop lines are plotted near the peaks of each segment and analysed for flow vortices at various heights. The characteristics of an 
individual $\mathrm{C}_{\mathrm{m}}$ plot can be better understood when we look at the blade vortex generation and interaction.

Figure 21 represents the z-vorticity contours of a $90^{\circ}$ helical blade VAWT operating at TSR 2.7 (Figure 21a-c), TSR 3.1 (Figure 21d-f), and TSR 3.5 (Figure 21g-i) at three different azimuth angles $\left(100^{\circ}, 130^{\circ}\right.$, and $\left.150^{\circ}\right)$ of rotation of Blade 1 . The colour notation used for the blades in Figures 17-19 is used here as well. It can be seen that the flow separation happens in lower tip speed ratio there by increasing the interaction of the shed vortex on the receding blades. This substantiates the observation about the secondary peaks found in Figure 20. The flow separations are found to be minimal in TSR3.5, thereby producing better $C_{m}$ when compared to other TSRs. It is also found that the z-vorticity generated by the shaft reduces as the TSR increases.

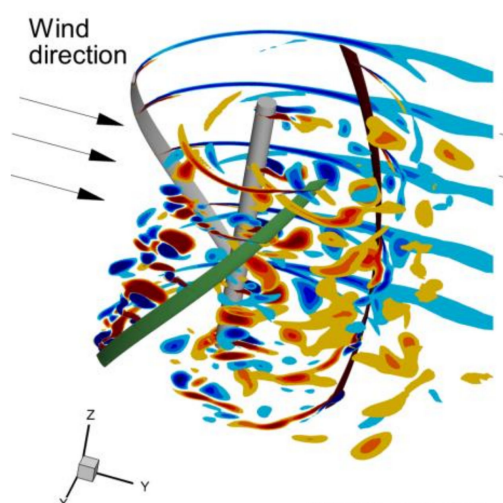

(a) $\theta=100 \mathrm{deg}$

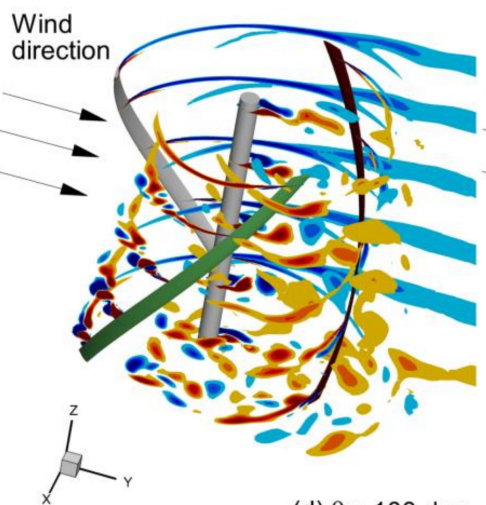

(d) $\theta=100 \mathrm{deg}$

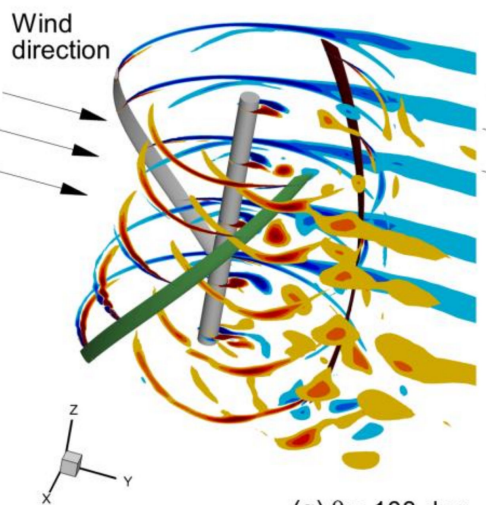

(g) $\theta=100 \mathrm{deg}$

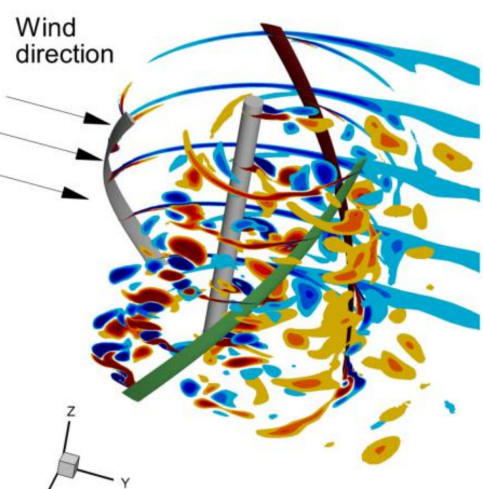

(b) $\theta=130 \mathrm{deg}$

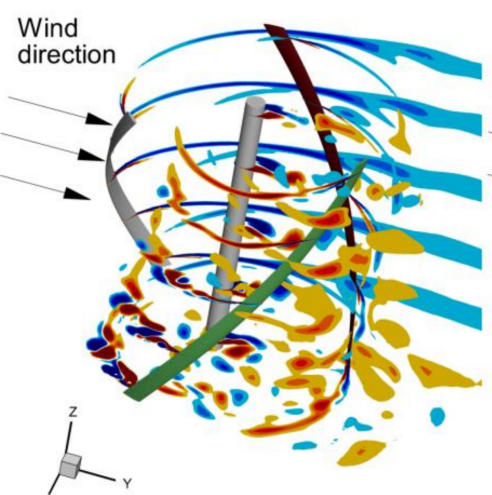

(e) $\theta=130 \mathrm{deg}$

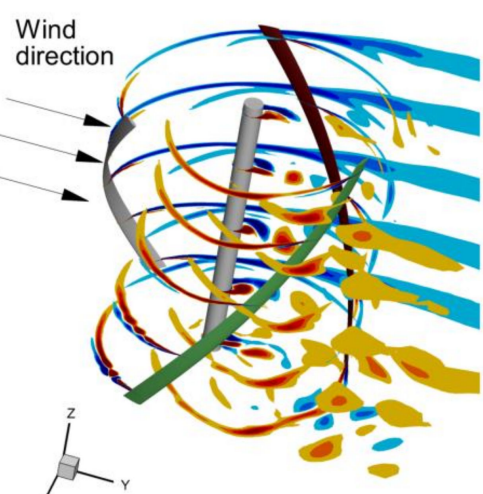

(h) $\theta=130 \mathrm{deg}$

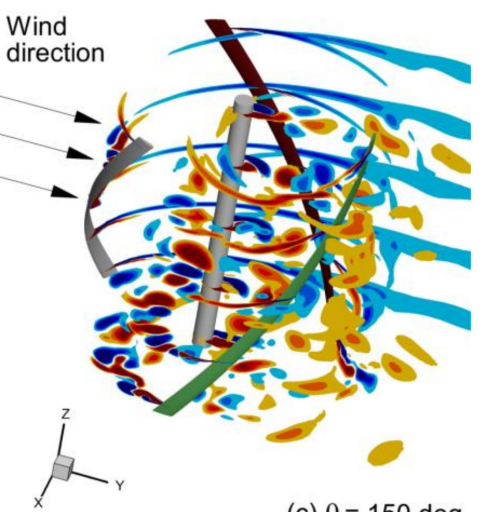

(c) $\theta=150 \mathrm{deg}$

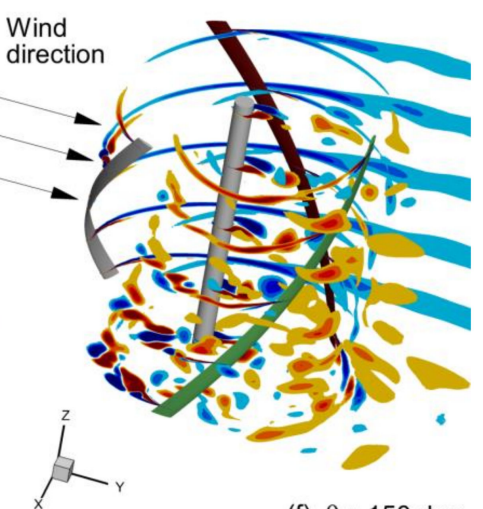

(f) $\theta=150 \mathrm{deg}$

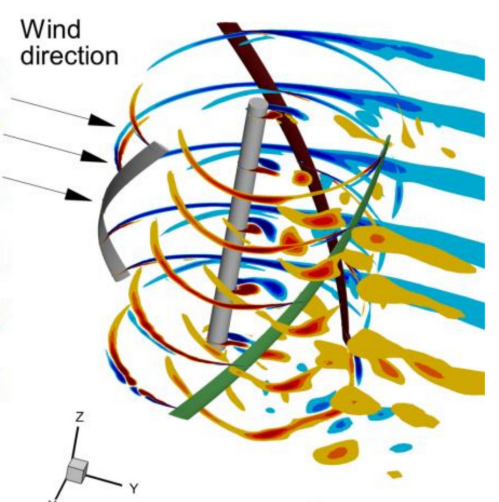

(i) $\theta=150 \mathrm{deg}$

Figure 21. Z-vorticity magnitude of $90^{\circ}$ helical-bladed VAWT at $100^{\circ}, 130^{\circ}$, and $150^{\circ}$ of azimuth angles of rotation of a turbine operating at $(\mathbf{a}-\mathbf{c})$ TSR $=2.7,(\mathbf{d}-\mathbf{f})$ TSR $=3.1$, and $(\mathbf{g}-\mathbf{i})$ TSR $=3.5$. 


\subsection{Effect of Helix Angle on Turbine Wake}

Figures 22 and 23 presents the time-averaged (over the last turbine revolution) normalised stream-wise velocity along the non-dimensionalised lateral line, $-2 \leq X / D \leq 2$, at different downstream locations in the turbine wake with $\mathrm{Y} / \mathrm{D}=2.0,3.0$, and 4.0 , for $\lambda=3.1$ (Figure 22) and 3.5 (Figure 23). The scaling of the wake was followed based on the work done by Kadum et al. [47] The following points are observed:

- The turbine wake gets weaker at TSR 3.1 for $120^{\circ}$ helical-bladed VAWT when compared with straight and other helical turbines.

- The wake profile gets weaker when the helix angle is increased even from a closer $\mathrm{X} / \mathrm{D}$ rage of 2 .

$\psi$

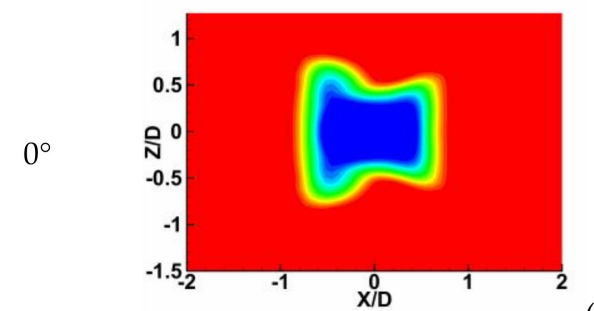

(a)

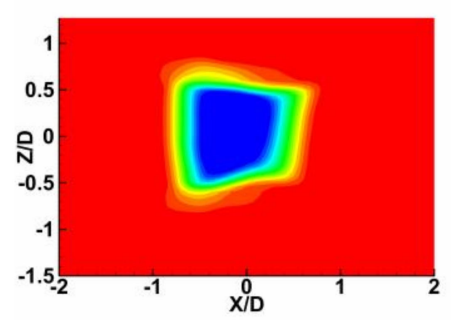

(d)

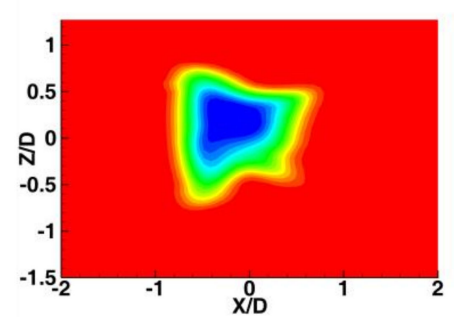

(g)

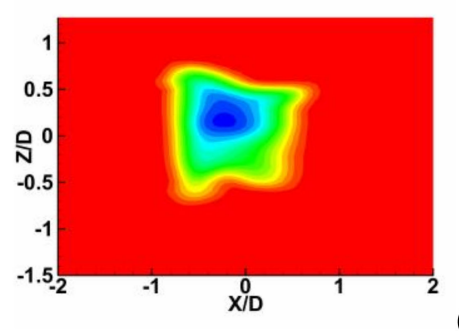

$\mathrm{Y} / \mathrm{D}=3$

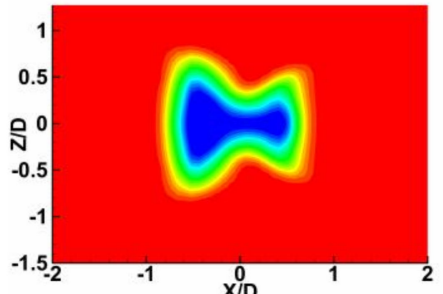

(b)

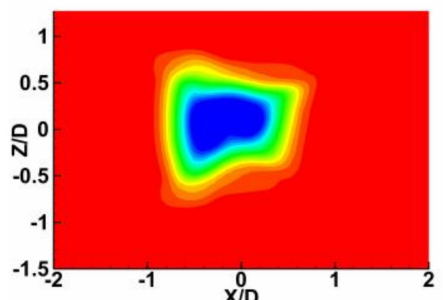

(e)

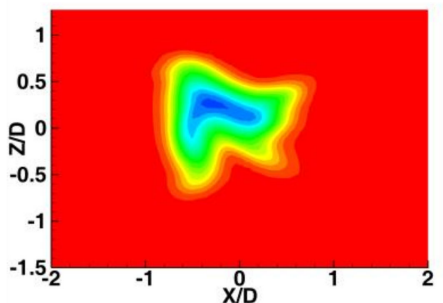

(h)

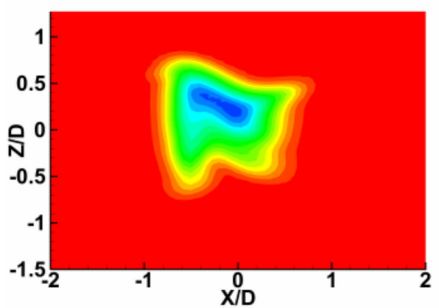

(k)
$\mathrm{Y} / \mathrm{D}=4$

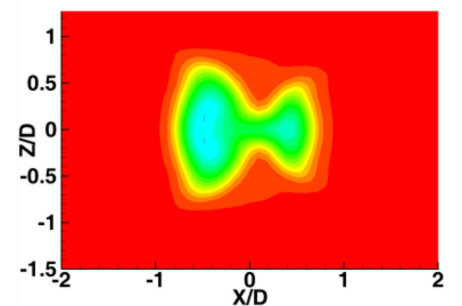

(c)

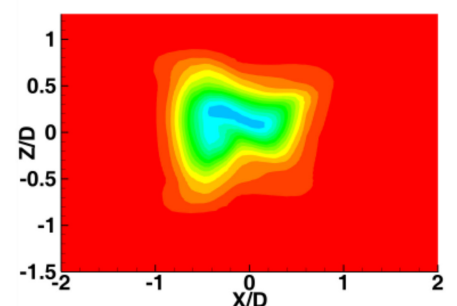

(f)

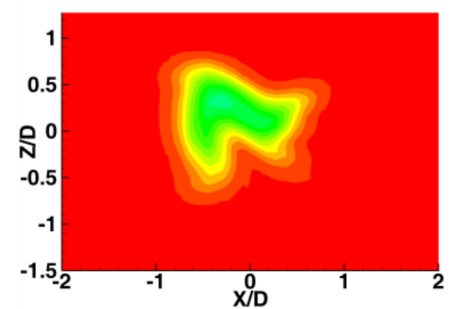

(i)

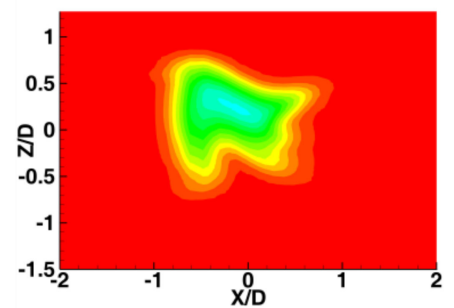

(1)

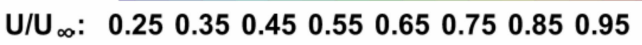

Figure 22. Wake structure of helical blade VAWT at $Y / D$ of $2(\mathbf{a}, \mathbf{d}, \mathbf{g}, \mathbf{j}), 3(\mathbf{b}, \mathbf{e}, \mathbf{h}, \mathbf{k})$, and $4(\mathbf{c}, \mathbf{f}, \mathbf{i}, \mathbf{l})$. For straight and helicalbladed VAWT at TSR $=3.1$. 
$60^{\circ}$

(a)

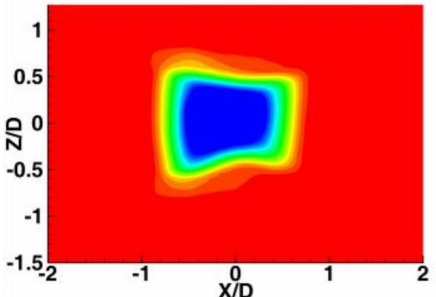

(d)
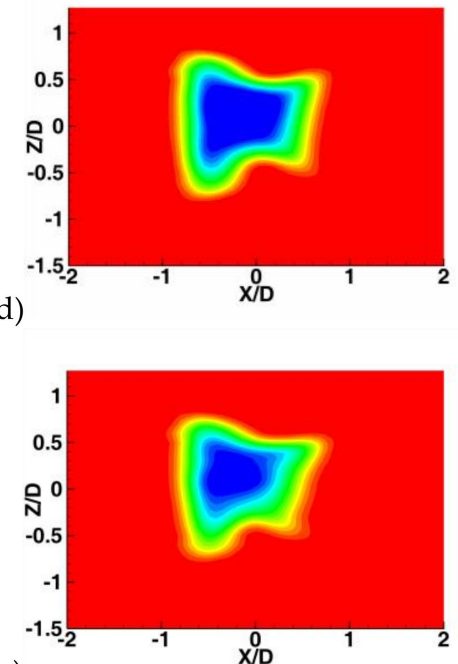

(g)

$\mathrm{U} / \mathrm{U}_{\infty}: \quad 0.250 .350 .450 .550 .650 .750 .850 .95$
$\mathrm{Y} / \mathrm{D}=3$

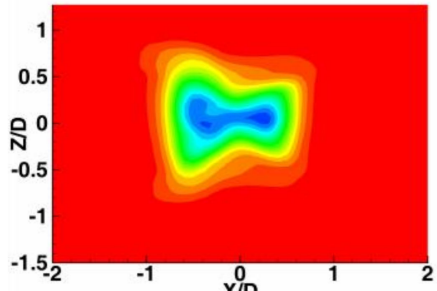

(b)

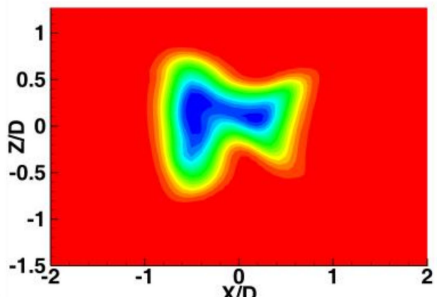

(e)

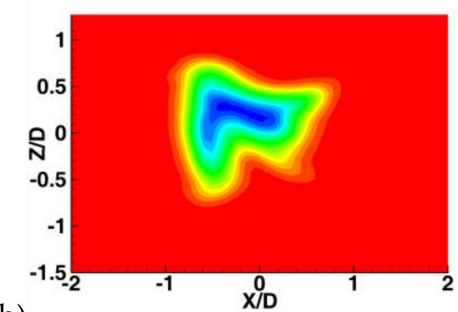

(h)

(i)

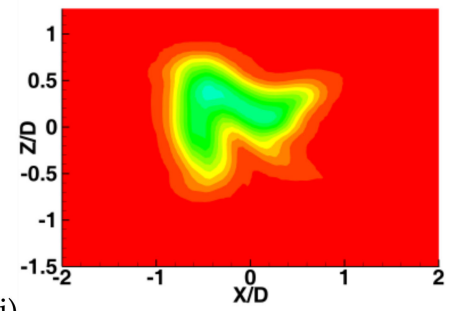

Figure 23. Wake structure of helical blade VAWT at Y/D of $2(\mathbf{a}, \mathbf{d}, \mathbf{g}), 3(\mathbf{b}, \mathbf{e}, \mathbf{h})$, and 4 (c,f,i). For helical-bladed VAWT at $\mathrm{TSR}=3.5$.

The wake of a helical-bladed VAWT unlike that with straight blades seems to be dissipating quickly as the helix angle increases. It further degrades when the tip speed ratio increases.

The turbine wake was studied in order to understand how the flow is going past the turbine. It is interesting to see how the wake dissipates over the length. Hence, the wake characteristic for a $90^{\circ}$ helical blade VAWT is studied to understand the wake better. Figure 24 indicated the normalised wake characteristics for a $90^{\circ}$ helical blade VAWT operating at TSR $=2.7,3.1$, and 3.5. This study is essential to understand how the flow past the turbine will behave, as it also gives idea on how much residual energy is left. The wake for a 3.9 TSR at a Y/D of 2, in comparison with other TSR, seems to have lost a lot of energy. It is also noted that the wake characteristic shapes remain almost similar for a given $\mathrm{Y} / \mathrm{D}$ despite different TSR. 
TSR

$\mathrm{Y} / \mathrm{D}=2$

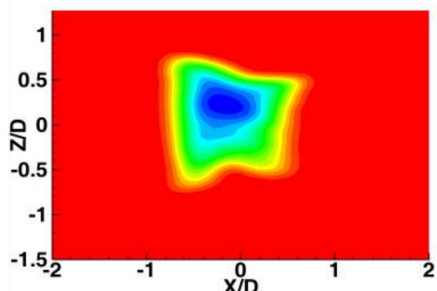

(a)

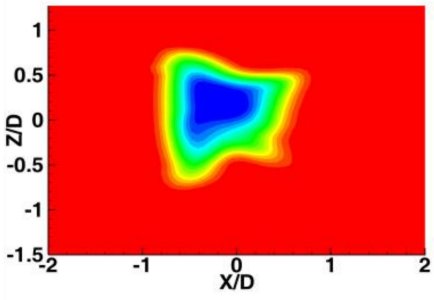

(d)

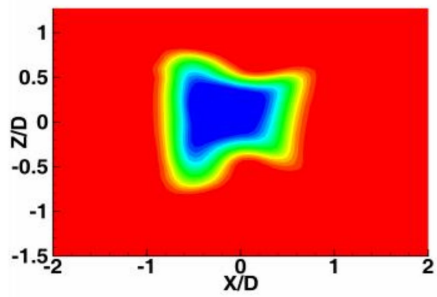

(g)

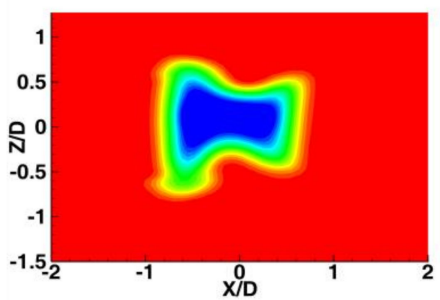

(j)
$\mathrm{Y} / \mathrm{D}=3$

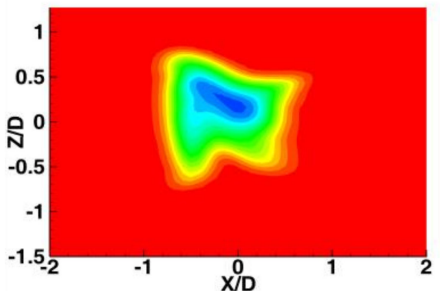

(b)

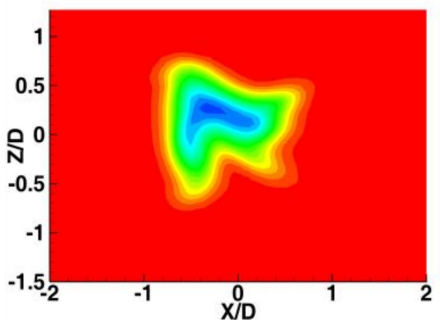

(e)

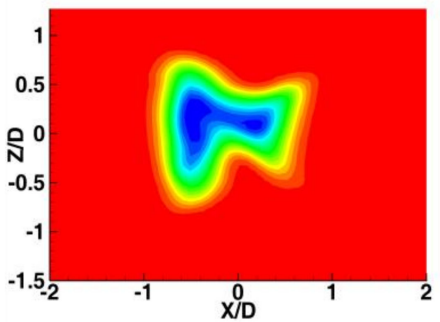

(h)

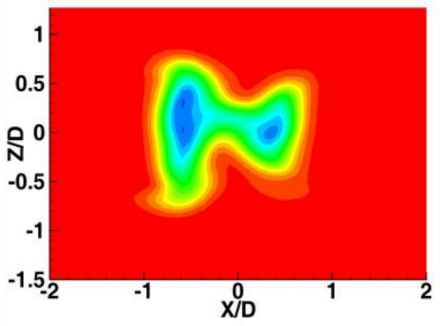

(k)
$\mathrm{Y} / \mathrm{D}=4$

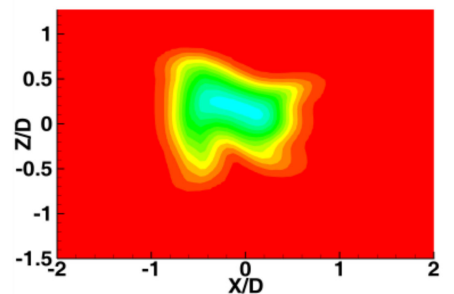

(c)

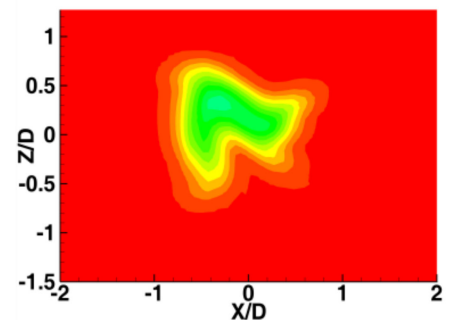

(f)

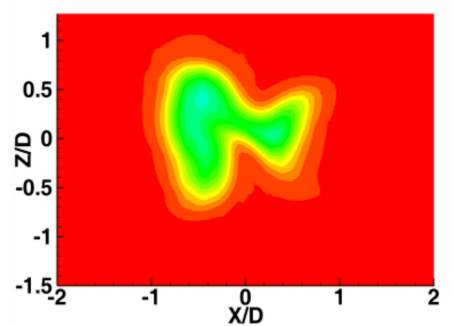

(i)

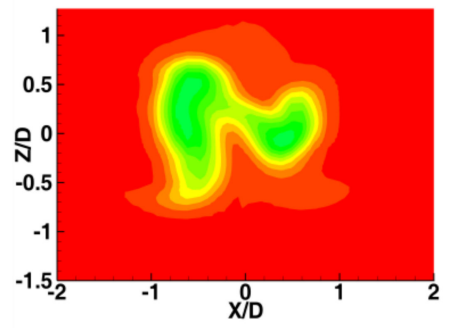

(1)

$\mathrm{U} / \mathrm{U}_{\infty}: \quad 0.250 .350 .450 .550 .650 .750 .850 .95$

(m)

Figure 24. Wake structure for a $90^{\circ}$ helical-bladed VAWT at $Y / D$ of $2(\mathbf{a}, \mathbf{d}, \mathbf{g}, \mathbf{j}), 3(\mathbf{b}, \mathbf{e}, \mathbf{h}, \mathbf{k})$, and 4 (c, Figure $2.7(\mathbf{a}-\mathbf{c}), 3.1$ (d-f), $3.5(\mathbf{g}-\mathbf{i})$ and $3.9(\mathbf{j}-\mathbf{1})$.

\section{Conclusions}

In the present study, the effect of helix angles varying from $60^{\circ}$ to $90^{\circ}$ for helical VAWT has been studied. The turbine diameter is $2.7 \mathrm{~m}$ and the height is $3 \mathrm{~m}$. A 3D CFD model has been used to analyse the performance of these turbines. The following are the major conclusions derived from the work

- The study on effect of helix angle of a vertical axis wind turbine has shown that the performance, wake interaction, and the flow interaction are different from those of a straight blade VAWT. 
- The performance of a $60^{\circ}$ helical blade VAWT was found to be outperforming all the other VAWT blade shapes and had a peak at a moderate TSR. However, it had the highest standard deviation from the average $C_{m}$ produced by the turbine blade. Normalised $C_{m}$ plots will enable understanding the moment coefficient characteristics.

- Analysing the quartile performance of the helical turbines gave more insights on how the power production was distributed across the azimuth angle of rotation. The variation in the quartile performance is backed by the standard deviation plot, which suggests that the minimum deviation is for $120^{\circ}$ helical-bladed VAWT, since the power production capabilities are spread across the quartiles

- An essential detailed analysis of loads on the sections of blades revealed that the leading segment, mid segment, and trailing segment of a single helical blade contributed different percentages of $\mathrm{Cm}$ to the cumulative blade $\mathrm{Cm}$. Z-vorticity contours at different heights of the turbine showed that the flow interference caused secondary peaks and also lead to better understanding of when and where the flow separation on the blade happens.

- In order to better understand the energy extraction, a wake analysis was performed, leading to the understanding that wakes dissipate quickly for non-straight blade VAWTs.

- Analysis of the effect of TSR on the performance of the turbine was also done with the help of a $90^{\circ}$ helical-bladed VAWT. Z-vorticity kept reducing as the TSR increased from 2.7 to 3.5 .

Author Contributions: Conceptualisation, R.K.V. and U.D.; methodology, R.K.V., U.D.; software, R.K.V., A.M.; validation, R.K.V. and U.D.; formal analysis, R.K.V.; investigation, R.K.V., A.R.; resources, A.M.; data curation, U.D.; writing —original draft preparation, U.D.; writing—review and editing, R.K.V., A.R.; visualisation, U.D.; supervision, R.K.V. and A.R. All authors have read and agreed to the published version of the manuscript.

Funding: This research received no external funding.

Acknowledgments: The authors would like to acknowledge the administration of Amrita Vishwa Vidyapeetham and King Abdul Aziz University of Science and Technology for the support extended for this work.

Conflicts of Interest: The authors declare no conflict of interest.

\section{References}

1. Streck, C.; Keenlyside, P.; Von Unger, M. The Paris Agreement: A new beginning. J. Eur. Environ. Plan. Law 2016, 13, 3-29. [CrossRef]

2. Agreement, P. Report of The Conference of The Parties on Its Twenty-First Session, Held in Paris from 30 November to 13 December 2015; FCCC/CP/2015/10/Add. 1; United Nations: New York, NY, USA; European Commision Secretariat: Bruxelles, Belgium, 2015.

3. Savaresi, A. The Paris agreement: A new beginning? J. Energy Nat. Resour. Law 2016, 13, 3-29. [CrossRef]

4. Delarue, E.D.; Luickx, P.J.; D'haeseleer, W.D. The actual effect of wind power on overall electricity generation costs and $\mathrm{CO}_{2}$ emissions. Energy Convers. Manag. 2009, 50, 1450-1456. [CrossRef]

5. Kirthika, N.; Ramachandran, K.I.; Kottayil, S.K. Deep Quantile Regression Based Wind Generation and Demand Forecasts. In Advances in Intelligent Systems and Computing, Proceedings of the International Conference on Soft Computing and Pattern Recognition, Hyderabad, India, 13-15 December 2019; Abraham, A., Jabbar, M., Tiwari, S., Jesus, I., Eds.; Springer: Cham, Swizerland, 2019; pp. 112-122.

6. Pedersen, E.; Persson Waye, K. Perception and annoyance due to wind turbine noise-a dose-response relationship. J. Acoust. Soc. Am. 2004, 116, 3460-3470. [CrossRef] [PubMed]

7. Wagner, S.; Bareiss, R.; Guidati, G. Wind Turbine Noise; Springer Science \& Business Media: Berlin/Heidelberg, Germany, 2012; ISBN 3642887104.

8. Crockford, N.J. A Review of the Possible Impacts of Wind Farms on Birds and Other Wildlife; Joint Nature Conservation Committee: Peterborough, UK, 1992.

9. Thönnißen, F.; Marnett, M.; Roidl, B.; Schröder, W. A Numerical Analysis to Evaluate Betz's Law for Vertical Axis Wind Turbines; IOP Publishing: Briatol, UK, 2016; Volume 753, p. 22056.

10. Madsen, H.A.; Paulsen, U.S.; Vitae, L. Analysis of VAWT Aerodynamics and Design Using the Actuator Cylinder Flow Model; IOP Publishing: Bristol, UK, 2014; Volume 555, p. 12065.

11. Gulve, P.; Barve, S.B. Design and construction of vertical axis wind turbine. Int. J. Mech. Eng. Technol. 2014, 5, 148-155. 
12. Tjiu, W.; Marnoto, T.; Mat, S.; Ruslan, M.H.; Sopian, K. Darrieus vertical axis wind turbine for power generation II: Challenges in HAWT and the opportunity of multi-megawatt Darrieus VAWT development. Renew. Energy 2015, 75, 560-571. [CrossRef]

13. Li, Q.; Maeda, T.; Kamada, Y.; Shimizu, K.; Ogasawara, T.; Nakai, A.; Kasuya, T. Effect of rotor aspect ratio and solidity on a straight-bladed vertical axis wind turbine in three-dimensional analysis by the panel method. Energy 2017, 121, 1-9. [CrossRef]

14. Li, Q.; Maeda, T.; Kamada, Y.; Murata, J.; Shimizu, K.; Ogasawara, T.; Nakai, A.; Kasuya, T. Effect of solidity on aerodynamic forces around straight-bladed vertical axis wind turbine by wind tunnel experiments (depending on number of blades). Renew. Energy 2016, 96, 928-939. [CrossRef]

15. Eboibi, O.; Danao, L.A.M.; Howell, R.J. Experimental investigation of the influence of solidity on the performance and flow field aerodynamics of vertical axis wind turbines at low Reynolds numbers. Renew. Energy 2016, 92, 474-483. [CrossRef]

16. Mantravadi, B.; Unnikrishnan, D.; Sriram, K.; Mohammad, A.; Vaitla, L.; Velamati, R.K. Effect of solidity and airfoil on the performance of vertical axis wind turbine under fluctuating wind conditions. Int. J. Green Energy 2019, 16. [CrossRef]

17. Rezaeiha, A.; Kalkman, I.; Blocken, B. Effect of pitch angle on power performance and aerodynamics of a vertical axis wind turbine. Appl. Energy 2017, 197, 132-150. [CrossRef]

18. El-Samanoudy, M.; Ghorab, A.A.E.; Youssef, S.Z. Effect of some design parameters on the performance of a Giromill vertical axis wind turbine. Ain Shams Eng. J. 2010, 1, 85-95. [CrossRef]

19. Chandramouli, S.; Premsai, T.P.; Prithviraj, P.; Mugundhan, V.; Velamati, R.K. Numerical analysis of effect of pitch angle on a small scale vertical axis wind turbine. Int. J. Renew. Energy Res. 2014, 4, 929-935.

20. Ferreira, C.S.; Geurts, B. Aerofoil optimization for vertical-axis wind turbines. Wind Energy 2015, 18, 1371-1385. [CrossRef]

21. Bedon, G.; De Betta, S.; Benini, E. Performance-optimized airfoil for Darrieus wind turbines. Renew. Energy 2016, 94, 328-340. [CrossRef]

22. Sengupta, A.R.; Biswas, A.; Gupta, R. Studies of some high solidity symmetrical and unsymmetrical blade H-Darrieus rotors with respect to starting characteristics, dynamic performances and flow physics in low wind streams. Renew. Energy 2016, 93, 536-547. [CrossRef]

23. Rezaeiha, A.; Kalkman, I.; Blocken, B. CFD simulation of a vertical axis wind turbine operating at a moderate tip speed ratio: Guidelines for minimum domain size and azimuthal increment. Renew. Energy 2017, 107, 373-385. [CrossRef]

24. Parker, C.M.; Leftwich, M.C. The effect of tip speed ratio on a vertical axis wind turbine at high Reynolds numbers. Exp. Fluids 2016, 57, 74. [CrossRef]

25. Siddiqui, M.S.; Rasheed, A.; Kvamsdal, T.; Tabib, M. Effect of turbulence intensity on the performance of an offshore vertical axis wind turbine. Energy Procedia 2015, 80, 312-320. [CrossRef]

26. Wekesa, D.W.; Wang, C.; Wei, Y.; Zhu, W. Experimental and numerical study of turbulence effect on aerodynamic performance of a small-scale vertical axis wind turbine. J. Wind Eng. Ind. Aerodyn. 2016, 157, 1-14. [CrossRef]

27. Bhargav, M.; Kishore, V.R.; Laxman, V. Influence of fluctuating wind conditions on vertical axis wind turbine using a threedimensional CFD model. J. Wind Eng. Ind. Aerodyn. 2016, 158, 98-108. [CrossRef]

28. Wang, Z.; Wang, Y.; Zhuang, M. Improvement of the aerodynamic performance of vertical axis wind turbines with leading-edge serrations and helical blades using CFD and Taguchi method. Energy Convers. Manag. 2018, 177, 107-121. [CrossRef]

29. Battisti, L.; Brighenti, A.; Benini, E.; Castelli, M.R. Analysis of Different Blade Architectures on Small VAWT Performance; IOP Publishing: Bristol, UK, 2016; Volume 753, p. 62009.

30. Han, D.; Heo, Y.G.; Choi, N.J.; Nam, S.H.; Choi, K.H.; Kim, K.C. Design, fabrication, and performance test of a 100-w helical-blade vertical-axis wind turbine at low tip-speed ratio. Energies 2018, 11, 1517. [CrossRef]

31. Battisti, L.; Persico, G.; Dossena, V.; Paradiso, B.; Castelli, M.R.; Brighenti, A.; Benini, E. Experimental benchmark data for H-shaped and troposkien VAWT architectures. Renew. Energy 2018, 125, 425-444. [CrossRef]

32. Bedon, G.; Castelli, M.R.; Benini, E. Proposal for an innovative chord distribution in the Troposkien vertical axis wind turbine concept. Energy 2014, 66, 689-698. [CrossRef]

33. Bedon, G.; Castelli, M.R.; Benini, E. Optimal spanwise chord and thickness distribution for a Troposkien Darrieus wind turbine. J. Wind Eng. Ind. Aerodyn. 2014, 125, 13-21. [CrossRef]

34. Persico, G.; Dossena, V.; Paradiso, B.; Battisti, L.; Brighenti, A.; Benini, E. Time-resolved experimental characterization of the wakes shed by $\mathrm{H}$-shaped and troposkien vertical axis wind turbines. J. Energy Resour. Technol. 2017, 139, 31203. [CrossRef]

35. Divakaran, U.; Kishore, V.R.; Ramesh, A. Effect of wind speed on the performance of Troposkein vertical axis wind turbine. Int. J. Renew. Energy Res. 2019, 9, 1510-1521.

36. Tjiu, W.; Marnoto, T.; Mat, S.; Ruslan, M.H.; Sopian, K. Darrieus vertical axis wind turbine for power generation I: Assessment of Darrieus VAWT configurations. Renew. Energy 2015, 75, 50-67.

37. Bhuyan, S.; Biswas, A. Investigations on self-starting and performance characteristics of simple $\mathrm{H}$ and hybrid $\mathrm{H}$-Savonius vertical axis wind rotors. Energy Convers. Manag. 2014, 87, 859-867. [CrossRef]

38. Lee, Y.-T.; Lim, H.-C. Numerical study of the aerodynamic performance of a $500 \mathrm{~W}$ Darrieus-type vertical-axis wind turbine. Renew. Energy 2015, 83, 407-415. [CrossRef]

39. Scheurish, F.; Fletcher, T.M.; Brown, R.E. The Influence of Blade Curvature and Helical Blade Twist on the Performance of a Vertical-Axis Wind Turbine. In Proceedings of the 48th AIAA Aerospace Sciences Meeting Including the New Horizons Forum and Aerospace Exposition, Orlando, FL, USA, 4-7 January 2010. 
40. Karimian, S.M.H.; Abdolahifar, A. Performance investigation of a new Darrieus Vertical Axis Wind Turbine. Energy 2020, 191, 116551. [CrossRef]

41. Bachant, P.; Wosnik, M. Performance measurements of cylindrical- and spherical-helical cross-flow marine hydrokinetic turbines, with estimates of exergy efficiency. Renew. Energy 2015, 74, 318-325. [CrossRef]

42. Marsh, P.; Ranmuthugala, D.; Penesis, I.; Thomas, G. Numerical investigation of the influence of blade helicity on the performance characteristics of vertical axis tidal turbines. Renew. Energy 2015, 81, 926-935. [CrossRef]

43. Pongduang, S.; Kayankannavee, C.; Tiaple, Y. Experimental Investigation of Helical Tidal Turbine Characteristics with Different Twists. Energy Procedia 2015, 79, 409-414. [CrossRef]

44. Rezaeiha, A.; Montazeri, H.; Blocken, B. On the accuracy of turbulence models for CFD simulations of vertical axis wind turbines. Energy 2019, 180, 838-857. [CrossRef]

45. Ansys ${ }^{\circledR}$. Academic Research FLUENT, Release 19.3, Help System, Fluent Theory Manual; ANSYS, Inc.: Canonsburg, PA, USA, 2020.

46. Mclaren, K.W. A Numerical and Experimental Study of Unsteady Loading of High Solidity Vertical Axis Wind Turbines. Ph.D. Thesis, McMaster University, Hamilton, ON, Canada, 2011.

47. Kadum, H.; Friedman, S.; Camp, E.H.; Cal, R.B. Development and scaling of a vertical axis wind turbine wake. J. Wind Eng. Ind. Aerodyn. 2018, 174, 303-311. [CrossRef] 